\title{
Quercetin Ameliorates Fatty Acid Oxidation in the Pathogenesis of NAFLD Based on Network Pharmacology and Molecular Docking
}

\section{Wenyu Zhu}

Chengdu University of Traditional Chinese Medicine

\section{Yufan Dai}

Chengdu University of Traditional Chinese Medicine

Jingjing Lin

Chengdu University of Traditional Chinese Medicine

Yanhong Chen

Chengdu University of Traditional Chinese Medicine

\section{Xiangshu Rao}

Chengdu University of Traditional Chinese Medicine

\section{Jiajun Wang}

Chengdu University of Traditional Chinese Medicine

Jiaxuan Zhou

Siemens PLM Software, Chengdu

Tao Shen ( $964515031 @ q q . c o m$ )

Chengdu University of Traditional Chinese Medicine

\section{Research Article}

Keywords: NAFLD, network pharmacology, fatty acid oxidation, quercetin, molecular docking

Posted Date: February 25th, 2021

DOI: https://doi.org/10.21203/rs.3.rs-223444/v1

License: (1) (1) This work is licensed under a Creative Commons Attribution 4.0 International License. Read Full License 


\title{
Quercetin Ameliorates Fatty Acid Oxidation in the Pathogene- sis of NAFLD Based on Network Pharmacology and Molecular Docking
}

\author{
Wenyu Zhu ${ }^{1}$, Yufan Dai ${ }^{1}$, Jingjing Lin ${ }^{1}$, Yanhong Chen ${ }^{1}$, Xiangshu Rao ${ }^{1}$, Jiajun Wang ${ }^{1}$, Jiaxuan Zhou ${ }^{2}$ and Tao \\ Shen 1 , * \\ 1 Chengdu University of Traditional Chinese Medicine, Chengdu, China; zwy1993@live.com (W.Z.); 764197682@qq.com (Y. D.); \\ 1011352034@qq.com (J.L.); 1779720172@qq.com (Y. C.); 2065239824@qq.com (X. R.); 526632532@qq.com (J. W.) \\ 2 Siemens PLM Software, Chengdu, China; zjx1994@live.com \\ * Correspondence: 964515031@qq.com; Tel.: +86-13980005243
}

\begin{abstract}
The high-fat diet (HFD) has dramatically increased the prevalence of non-alcoholic fatty liver disease (NAFLD), such that it is the leading cause of chronic liver disease worldwide. With novel knowledge on pathogenesis of NAFLD, we illustrated molecular targets for pharmacologic and experimental approaches. Our study showed that quercetin was the valuable drug candidate, and the core targets such as PPAR $\alpha$, MCAD, LCAD, CPT1-L, CPT2 and FATP2 which participate in fatty acid oxidation were selected to perform further in vivo experiments based on network pharmacology and molecular docking technology. The effect was investigated in a high-fat diet-induced NAFLD male Sprague Dawley rat model by hepatic biochemical assays. Serum alanine aminotransferase, aspartate aminotransferase, alkaline phosphatase, and $\gamma$-glutamyl transferase analyses assessed liver function. Liver tissues were collected for histological staining, quantitative real-time PCR and western blotting. Greatly, quercetin reduced hepatic lipid accumulation and inflammation, ameliorated pathological liver changes, and up-regulated the expression levels of hepatic effectors in fatty acid oxidation. The results suggest the potential of quercetin as a nutritional supplement to HFD-induced NAFLD. Furthermore, the antagonist and agonist of PPAR $\alpha$ provided a reliable scientific basis that these above-mentioned effectors were activated by quercetin via the PPAR pathway.
\end{abstract}

Keywords: NAFLD; network pharmacology; fatty acid oxidation; quercetin; molecular docking

\section{Introduction}

Non-alcoholic fatty liver disease (NAFLD) is defined as the accumulation of excessive fat in more than $5 \%$ of hepatocytes without significant alcohol intake ${ }^{1}$. NAFLD is a progressive liver metabolic disease with a broad spectrum of histological abnormalities, ranging from simple steatosis to non-alcoholic steatohepatitis, cirrhosis, and even hepatocellular carcinoma 2,3 . NAFLD, especially non-alcoholic steatohepatitis, is often associated with type 2 diabetes mellitus, hyperlipidemia, and central obesity ${ }^{4}$. Liver diseases that progress to fibrosis and cirrhosis are irreversible, and the only option for treatment is liver transplantation, which is a substantial economic burden worldwide ${ }^{5}$. The liver is an essential metabolic organ that principally impacts fatty acid $\beta$-oxidation (FAO), lipid catabolism and other physiological processes, such as glucose homeostasis and adipocyte differentiation ${ }^{6}$. In the setting of overnutrition, hepatic fatty acid metabolism is altered, the excessive triglyceride within hepatocytes aggravates chronic inflammatory response and metabolic disturbance ${ }^{7,8}$. Despite advances in this field, the molecular mechanisms of NAFLD remain obscure.

From the perspective of traditional Chinese medicine, improper diet adversely affects the spleen and stomach, leading to dysfunction of transport and transformation. The food cannot be transformed into nutrients, then turbid phlegm dampness consequently accumulates in the liver, which results in NAFLD. Coptidis Rhizoma, a plant from the Ranunculus family, affects the middle energizer by drying dampness and purging pathogenic fire ${ }^{9}$. Evodiae Fructus, a plant of the Rue family, specializes in the middle energizer. The two herbs are predominant pharmacological components of Zuojin pill 10,11, a prescription of Traditional Chinese Medicine and are widely used in digestive system diseases according to clinical studies, such as distention, fullness, swelling, and dysentery. These symptoms strongly correlate with the symptoms of NAFLD, providing a new approach for the treatment of NAFLD.

Network science offers new ideas to scientifically investigate the pathogenesis of diseases. Computational molecular docking is a technology based on experimental structural design and protein-protein interactions ${ }^{12}$. Network pharmacology is proved to be a promising method to understand herbal formulas and predict novel drugs or potential disease targets ${ }^{13}$. In this study, we firstly illustrated multi-target mechanisms of NAFLD based on system pharmacology analyses and then to verify the intersection protein levels using in vivo experiments by a high-fat diet-induced NAFLD Sprague Dawley rat model. Our study showed that quercetin was one valuable drug candidate, extracted from Coptidis 
Rhizoma and Evodiae Fructus in common for alleviating NAFLD. Based on the discovery, we aimed to utilize network 48 pharmacology and molecular docking to explore the association between quercetin and signal pathways in NAFLD.

\section{Results}

\subsection{Active compounds and targets screening}

Considering some correlative factors, 30 active compounds from Coptidis Rhizoma and Evodiae Fructus had OB $\geqslant$ $30 \%$ and DL $\geqslant 0.18$ were obtained with the help of the Traditional Chinese Medicine Systems Pharmacology Database and Analysis Platform (TCMSP, https://tcmspw.com/tcmsp.php/) ${ }^{14}$, shown in the Table 1.

Table 1 Active compounds from Coptidis Rhizoma and Evodiae Fructus and their corresponding predicted oral bioavailability (OB) and drug-likeliness (DL).

\begin{tabular}{|c|c|c|c|c|c|}
\hline No. & MOL ID & Source & Molecule Name & OB $(\%)$ & DL \\
\hline 1 & MOL002907 & Coptidis Rhizoma & Corchoroside A_qt & 104.95 & 0.78 \\
\hline 2 & MOL008647 & Coptidis Rhizoma & Moupinamide & 86.71 & 0.26 \\
\hline 3 & MOL003958 & Evodiae Fructus & Evodiamine & 86.02 & 0.64 \\
\hline 4 & MOL004018 & Evodiae Fructus & Goshuyuamide I & 83.19 & 0.39 \\
\hline 5 & MOL004014 & Evodiae Fructus & Evodiamide & 73.77 & 0.28 \\
\hline 6 & MOL003963 & Evodiae Fructus & hydroxyevodiamine & 72.11 & 0.71 \\
\hline 7 & MOL004019 & Evodiae Fructus & GoshuyuamideII & 69.11 & 0.43 \\
\hline 8 & MOL003942 & Evodiae Fructus & Rutaevine & 66.05 & 0.58 \\
\hline 9 & MOL000785 & Coptidis Rhizoma & palmatine & 64.6 & 0.65 \\
\hline 10 & MOL004021 & Evodiae Fructus & Gravacridoneshlirine & 63.73 & 0.54 \\
\hline 11 & MOL000622 & Coptidis Rhizoma & Magnograndiolide & 63.71 & 0.19 \\
\hline 12 & MOL002903 & Coptidis Rhizoma & (R)-Canadine & 55.37 & 0.77 \\
\hline 13 & MOL004017 & Evodiae Fructus & Fordimine & 55.11 & 0.26 \\
\hline 14 & MOL000354 & Evodiae Fructus & isorhamnetin & 49.6 & 0.31 \\
\hline 15 & MOL003974 & Evodiae Fructus & Evocarpine & 48.66 & 0.36 \\
\hline 16 & MOL000098 & $\begin{array}{c}\text { Coptidis Rhizoma, } \\
\text { Evodiae Fructus }\end{array}$ & quercetin & 46.43 & 0.28 \\
\hline 17 & MOL002668 & Coptidis Rhizoma & Worenine & 45.83 & 0.87 \\
\hline 18 & MOL013352 & $\begin{array}{c}\text { Coptidis Rhizoma, } \\
\text { Evodiae Fructus }\end{array}$ & Obacunone & 43.29 & 0.77 \\
\hline 19 & MOL002897 & Coptidis Rhizoma & epiberberine & 43.09 & 0.78 \\
\hline 20 & MOL003956 & Evodiae Fructus & dihydrorutaecarpine & 42.27 & 0.6 \\
\hline 21 & MOL003943 & Evodiae Fructus & Rutalinidine & 40.89 & 0.22 \\
\hline 22 & MOL002662 & Evodiae Fructus & rutaecarpine & 40.3 & 0.6 \\
\hline 23 & MOL000358 & Evodiae Fructus & beta-sitosterol & 36.91 & 0.75 \\
\hline 24 & MOL000359 & Evodiae Fructus & sitosterol & 36.91 & 0.75 \\
\hline 25 & MOL001454 & $\begin{array}{c}\text { Coptidis Rhizoma, } \\
\text { Evodiae Fructus }\end{array}$ & berberine & 36.86 & 0.78 \\
\hline 26 & MOL002904 & Coptidis Rhizoma & Berlambine & 36.68 & 0.82 \\
\hline 27 & MOL002894 & Coptidis Rhizoma & berberrubine & 35.74 & 0.73 \\
\hline 28 & MOL000762 & Coptidis Rhizoma & Palmidin A & 35.36 & 0.65 \\
\hline 29 & MOL004020 & Evodiae Fructus & gossypetin & 35 & 0.31 \\
\hline 30 & MOL001458 & Coptidis Rhizoma & coptisine & 30.67 & 0.86 \\
\hline
\end{tabular}

After conversion into the UniProt Knowledgebase (UniProtKB, https://www.uniprot.org/), a total of 147 drug targets from the two herbs and 70 disease targets linked to NAFLD were screened from the TCMSP and DisGeNET database (https://www.disgenet.org/). Interestingly, it is shown that both Coptidis Rhizoma and Evodiae Fructus contain quercetin, one of the most abundant dietary flavonoids, and the beneficial effects of quercetin on lipid accumulation and antioxidation associated with NAFLD have been reported recently. However, the mechanisms of quercetin for NAFLD 
are poorly understood, for many reasons, quercetin was selected as the drug candidate to perform further experimental verification. The two-dimensional (2D) structure of quercetin is shown in Figure 1a.

\subsection{Protein-protein interaction (PPI) network analysis}

Our study showed that the proteins encoded by target genes had complex interactions, providing a basis for molecular docking technology. The physical interaction between proteins brings on biological signaling and other aspects of life processes under special environment. We imported the protein-protein interaction core network (PPICN) into Cytoscape for further analysis. Figure $1 \mathrm{~b}$ shows the 10 intersecting elements between herbs and NAFLD grouped by PPICN; furthermore, the predicted target proteins were harvested: transforming growth factor beta-1 proprotein (TGFB1), interleukin-4 (IL-4), interleukin-1 alpha (IL-1 $\alpha$ ), interstitial collagenase (MMP1), aryl hydrocarbon receptor (AHR), protein kinase C alpha type (PRKCA), cytochrome P450 1A2 (CYP1A2), glutathione S-transferase P (GSTP1), glutathione S-transferase Mu 1 (GSTM1) and peroxisome proliferator-activated receptor alpha (PPAR $\alpha$ ).

\subsection{Network construction analysis}

A drug-compound-gene-disease network was built using Cytoscape v3.8.0 software in Figure 1c. The edges indicate the nodes that can interact with each other. The two green nodes represent the two herbs. The purple node represents the disease exclusively. The 44 orange nodes represent all active compounds in Coptidis Rhizoma and Evodiae Fructus. The 206 cyan nodes represent the target genes of the drugs and the disease. The 10 blue nodes represent overlapping genes between the disease and drugs, and then the 10 common targets were predicted to be therapeutic targets for the treatment of NAFLD.<smiles>O=c1c(O)c(-c2ccc(O)c(O)c2)oc2cc(O)cc(O)c12</smiles>

a

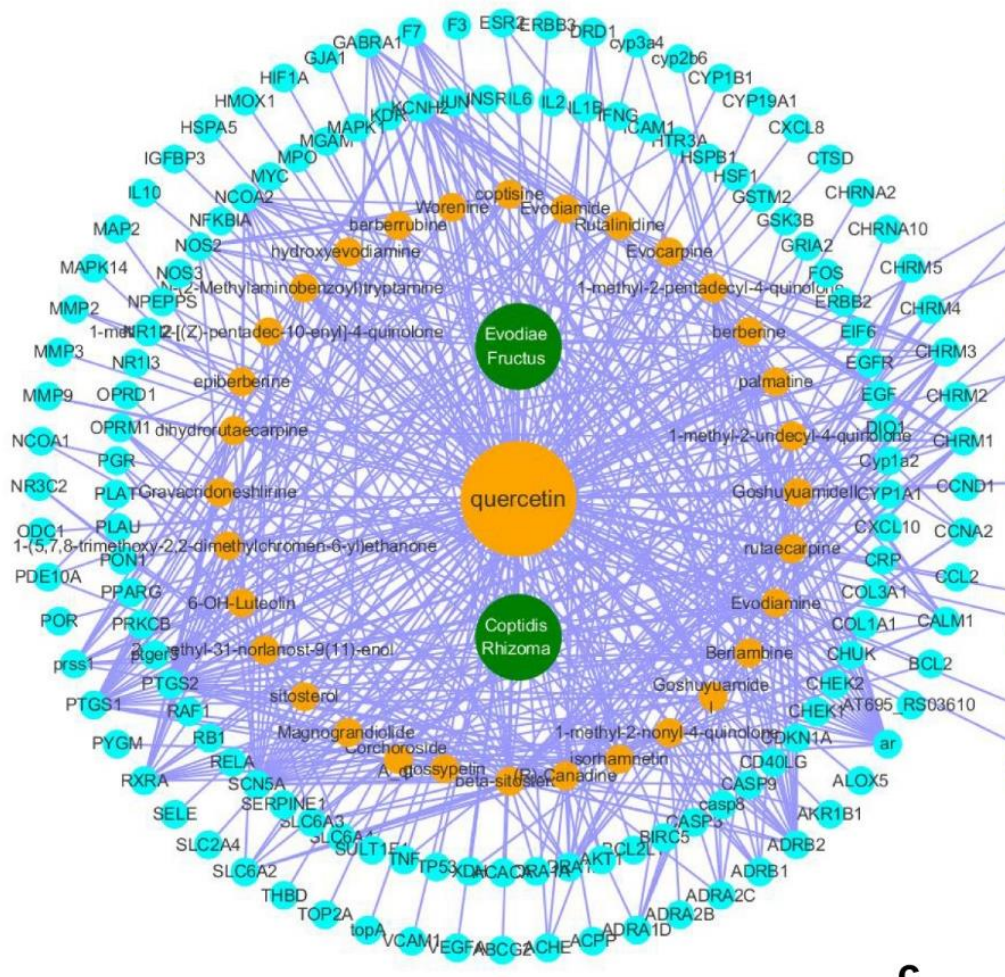

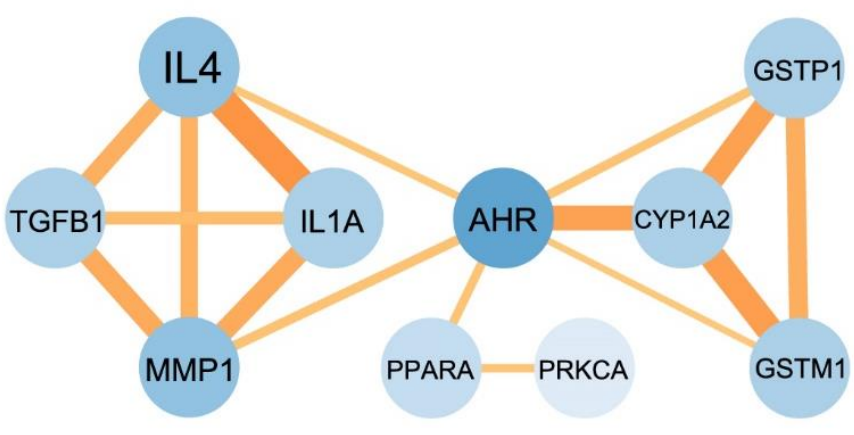

b

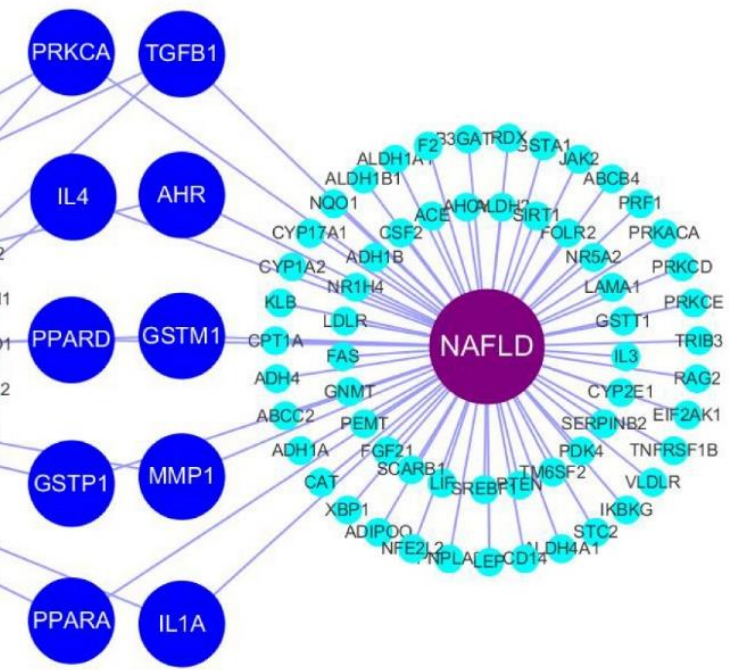

Figure 1 (a) The two-dimensional (2D) structure of quercetin. (b) The protein-protein interaction (PPI) network of proteins targeted by Coptidis Rhizoma and Evodiae Fructus and encoded by proteins associated with non-alcoholic fatty liver disease. (c) The potential drug-compound-gene-disease network. 
2.4. Gene Ontology (GO) based functional enrichment and the Kyoto Encyclopedia of Genes and Genomes (KEGG) pathway enrichment analyses

GO terms and KEGG pathway enrichment analyses yielded 20 entries through the intersection targets $(\mathrm{P}<0.005)$, and the details are listed in Table 2. Notably, this study found that PPAR signaling pathway was associated with many potential functions indicated by GO and KEGG enrichment, such as positive regulation of transcription, RNA polymerase II transcription factor activity, ligand-activated sequence-specific DNA binding, and the intracellular receptor signaling pathway. Additionally, PPAR $\alpha$ was connected with other biological targets as a core protein derived from Coptidis Rhizoma and Evodiae Fructus, providing important evidence supporting treatment strategies. More importantly, PPAR $\alpha$ participates in regulation of fatty acid transport and oxidation, cholesterol metabolism and lipid transport in the NAFLD treatment.

Table 2 GO terms and KEGG pathway enrichment analyses.

\begin{tabular}{ccccc}
\hline Category & Term & Count & \% & P value \\
\hline GO: BP & GO:0045893 positive regulation of transcription, DNA-templated & 5 & 45.45 & 0.000159 \\
GO: MF & GO:0004879 RNA polymerase II transcription factor activity, ligand- & 3 & 27.27 & 0.000197 \\
& activated sequence-specific DNA binding & & & \\
GO: BP & GO:0030522 intracellular receptor signaling pathway & 3 & 27.27 & 0.000222 \\
GO: MF & GO:0019899 enzyme binding & 4 & 36.36 & 0.000824 \\
GO: MF & GO:0008144 drug binding & 3 & 27.27 & 0.000879 \\
GO: BP & GO:0006805 xenobiotic metabolic process & 3 & 27.27 & 0.000936 \\
KEGG & hsa05200:Pathways in cancer & 5 & 45.45 & 0.00105 \\
GO: BP & GO:0032355 response to estradiol & 3 & 27.27 & 0.001271 \\
GO: BP & GO:0045944 positive regulation of transcription from RNA polymer- & 5 & 45.45 & 0.00183 \\
& ase II promoter & & & \\
GO: BP & GO:0002248 connective tissue replacement involved in inflammatory & 2 & 18.18 & 0.00238 \\
& response wound healing & & & \\
GO: BP & GO:0071677 positive regulation of mononuclear cell migration & 2 & 18.18 & 0.00238 \\
GO: BP & GO:0045892 negative regulation of transcription, DNA-templated & 4 & 36.36 & 0.002678 \\
KEGG & hsa05321:Inflammatory bowel disease (IBD) & 3 & 27.27 & 0.002941 \\
KEGG & hsa03320:PPAR signaling pathway & 3 & 27.27 & 0.003219 \\
KEGG & hsa00982:Drug metabolism - cytochrome P450 & 3 & 27.27 & 0.003315 \\
KEGG & hsa05140:Leishmaniasis & 3 & 27.27 & 0.003608 \\
KEGG & hsa00980:Metabolism of xenobiotics by cytochrome P450 & 3 & 27.27 & 0.003914 \\
KEGG & hsa05204:Chemical carcinogenesis & 3 & 27.27 & 0.004561 \\
GO: MF & GO:0005125 cytokine activity & 3 & 27.27 & 0.004604 \\
GO: BP & GO:0002674 negative regulation of acute inflammatory response & 2 & 18.18 & 0.004755 \\
\hline
\end{tabular}

2.5. Molecular docking analysis

The 3D structures of PPAR $\alpha$ (PDB ID: 2REW), carnitine O-palmitoyltransferase 1 (CPT1-L) (PDB ID: 2LE3), medium-chain specific acyl-CoA dehydrogenase (MCAD) (PDB ID: 4P13) were downloaded from the RCSB PDB database (https://www.rcsb.org/). As a result, the docking scores of 2LE3, 2REW and 4P13 were -5.0, -8.4 and -9.3 and molecular docking analysis indicated that quercetin could easily enter and bind the active pocket of PPAR $\alpha$ and MCAD proteins by AutoDock Vina 1.1.2. However, the ligand of quercetin has less firm binding with CPT1-L receptor molecule. Figure 2 a shows the binding mode between the receptor protein 2REW and the small ligand molecule. The amino acid residues Tyr314, His440, and Gln277 form hydrogen bond interactions with the small ligand molecule. The amino acid residues Tyr464, Ile317, Phe318, Leu321, Cys276, Met355, Phe273, Ile354, Ile447 form hydrophobic interactions with small ligand molecules. Figure $2 \mathrm{~b}$ shows the binding mode between receptor protein $4 \mathrm{P} 13$ and small ligand molecules. Residues Glu376, Gly377, Thr168 form hydrogen bond interactions with small ligand molecules, and amino acid residues Tyr375, Thr378, Ile374, Trp166, Ile167, Thr136, Tyr133 form hydrophobic interactions with small ligand molecules. Figure 2c shows the binding mode between receptor protein 2LE3 and small ligand molecules. Amino acid residues Ile31 and Trp39 form hydrogen bond interactions with small ligand molecules, and amino acid residues Gly35 and Ser38 form hydrophobic interactions with small ligand molecules. (Figure 2) 

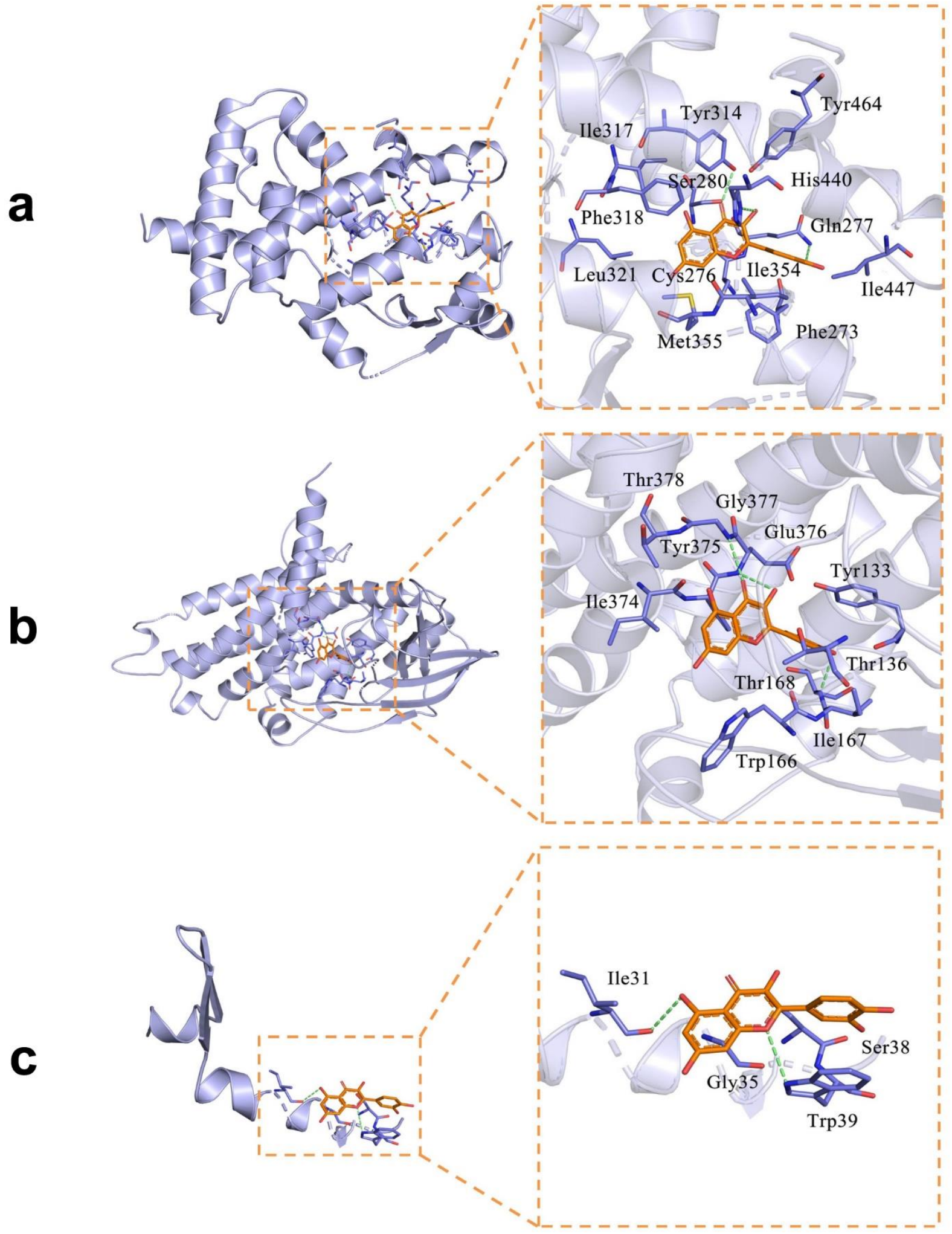

Figure 2 Molecular docking between the small molecule ligands of quercetin and 2REW (encoded by PPAR $\alpha$ ), 4P13 (encoded by MCAD), and 2LE3 (encoded by CPT1-L) protein receptors.

2.6. Effect of quercetin on body weight, epididymal fat weight and liver index

Compared with the Control group, the NAFLD groups showed an extraordinary increase in body weights before 12 weeks $(\mathrm{P}<0.01)$, and the indicator continued to grow in the high-fat diet (HFD) group until the 16th week $(\mathrm{P}<0.05)$. 
During the administration period, body weights declined sharply in quercetin at a high dose (Q-HD) group, fenofibrate (FF) group, and the FF + MK886 group when compared with those in the Q-HD + MK886 and the Control groups (P $<0.01$ ). (Figure 3a)

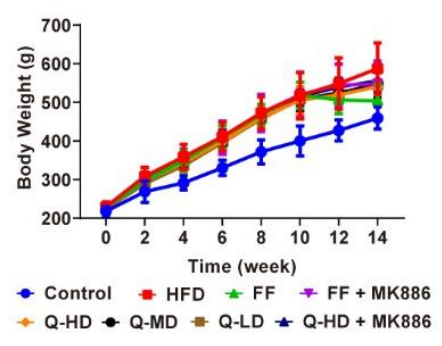

a
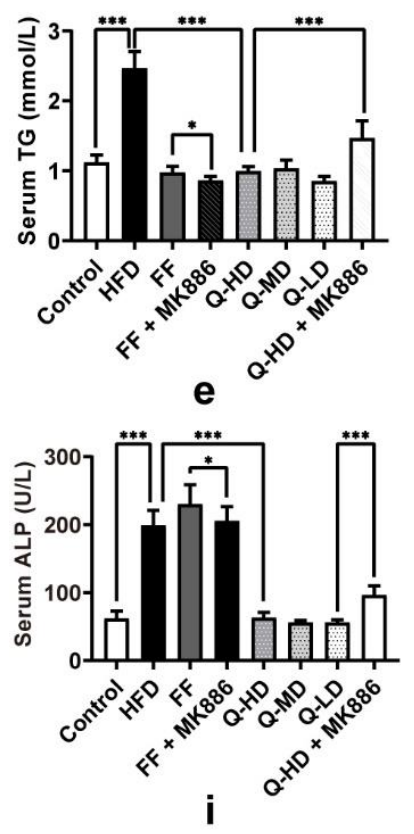

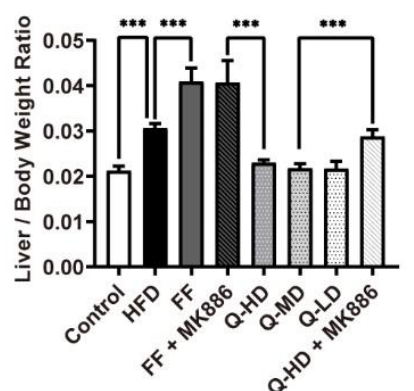

b
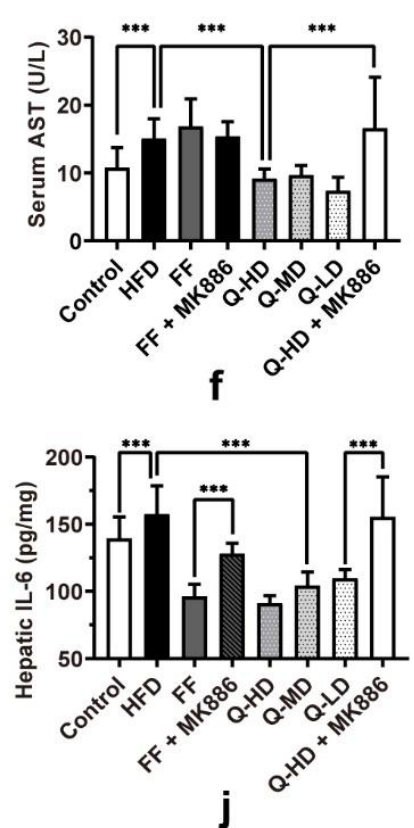
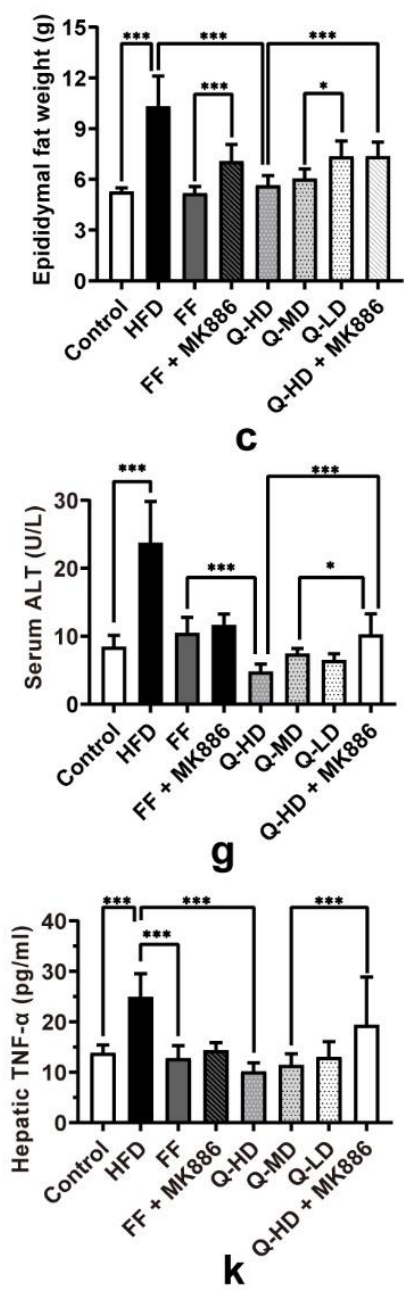

117

118

119

Figure 3 (a) Changes of body weight in different groups of rats. (b) The liver and body weight ratios (the liver indices) in different groups of rats. (c) The epididymal fat weights in different groups of rats. Two sensitive indices, the total cholesterol (d) and triglycerides (e), for quercetin effects on the lipid accumulation in the liver induced by a high-fat diet contents in liver tissue. The serum levels of aspartate aminotransferase (f), alanine aminotransferase (g), $\gamma$-glutamyl transferase (h) and alkaline phosphatase (i) in experimental groups. (j-I) Hepatic inflammatory factors levels of the tumor necrosis factor- $\alpha$ (TNF- $\alpha$ ), interleukin- 6 (IL-6) and interleukin-1 $\beta$ (IL-1 $\beta$ ) were compared among all groups. All results were expressed as the mean $\pm \mathrm{SD}, \mathrm{n}=10 . * \mathrm{P}<0.05, * * * \mathrm{P}<0.01$.

In Figure 3b, the liver and body weight ratios (the liver indices) in FF and FF + MK886 were significantly increased, whereas the three quercetin groups steadily reduced. Compared with the HFD group, there was no apparent decline in the Q-HD + MK886. However, no significant differences in the ratio were observed among the three groups treated with quercetin $(\mathrm{P}>0.05)$. In Figure $3 c$, the epididymal fat weight was notably increased in HFD group than the Control group. Compared with the HFD group, the epididymal fat weights were decreased in the Q-HD and FF groups, and were significantly reversed by MK886 ( $<<0.01)$.

\subsection{Quercetin ameliorated HFD-induced lipid accumulation}

As shown in Figure 4a, livers in the HFD group were found to be more yellow and greasy in appearance compared with the Control group. Furthermore, quercetin groups exhibited a reversal of this change to a certain extent compared with the MK886 group. Histological examination with oil red O (ORO) staining indicating hepatic steatosis revealed a dramatic increase in lipid accumulation in the HFD group $(72.01 \pm 4.62 \%)$ in contrast to the Control group $(1.28 \pm 0.26 \%)$ and the Q-HD group $(35.02 \pm 11.03 \%)$. The FF group $(6.61 \pm 7.24 \%)$ had significantly lower lipid accumulation than the $\mathrm{FF}+\mathrm{MK} 886$ group $(23.90 \pm 6.84 \%)$, and the ORO-positive staining in the Q-HD + MK886 group $(66.42 \pm 8.12 \%)$ was also higher than the Q-HD group. The percent area of ORO-staining in the quercetin at a medium dose (Q-MD) group (24.38 $\pm 7.65 \%$ ) was lower compared with the Q-HD and quercetin at a low dose (Q-LD) group (35.98 $\pm 12.94 \%)$. (Figure $4 \mathrm{~b})$ 
As shown in Figure 3d, e, the total cholesterol (TC) and triglycerides (TG) levels were decreased in the Q-HD, Q$\mathrm{MD}$, Q-LD, and FF groups compared with those in the HFD group $(\mathrm{P}<0.01)$. However, there was no statistical significance observed compared with the Control group. The serum TC levels of the two groups treated with MK886 were notably increased, and the FF + MK886 group was significantly higher than that of the HFD group. In the Q-HD + MK886 group, TG levels increased more than in the Q-HD group $(\mathrm{P}<0.01)$.

\subsection{Quercetin ameliorated liver function and reduced inflammation}

Serum alanine aminotransferase (ALT), aspartate aminotransferase (AST), alkaline phosphatase (ALP), and $\gamma$-glutamyl transferase $(\gamma-\mathrm{GT})$ are important targets that can indicate hepatic function damage. Compared with the Control group, all these biochemical indicators were increased in the HFD group. After 4-week-treatment with quercetin, all biochemical markers were remarkably decreased compared with the HFD group $(\mathrm{P}<0.01)$. Notably, ALP and AST activities were highest in the FF group $(\mathrm{P}<0.01)$. Moreover, MK886 caused a significant increase in AST, ALT, $\gamma$-GT, and ALP in the serum compared with the Q-HD + MK886 group ( $\mathrm{P}<0.01)$. The tumor necrosis factor- $\alpha$ (TNF- $\alpha$ ), interleukin6 (IL-6) and interleukin-1 $\beta$ (IL-1 $\beta$ ) levels in HFD livers were notably higher than those in the Control group and the quercetin groups exhibited a reduction of inflammatory factors in various degrees. The FF group had significantly lower hepatic levels of IL-6 and IL-1 $\beta$ than FF + MK886 group, but no significant differences in TNF- $\alpha$ level. Compared with the three quercetin groups, these hepatic inflammatory factors were significantly reversed by MK886. (Figure 3f-1)

Histopathological changes indicated by hematoxylin and eosin (HE) staining showed a small amount of lymphocyte infiltration with well-arranged hepatic lobules and no apparent fatty degeneration in the liver tissue after quercetin and fenofibrate intervention. In the HFD group, hepatic injury and fibrosis were observed with necrotic foci, increased eosinophilic cytoplasm, hepatocellular ballooning, and fibroblasts in liver tissues. Meanwhile, the degree of hepatocyte steatosis and lobular inflammation in the liver did not change in the two MK886 groups compared with the other treatment groups. (Figure 4c)

a

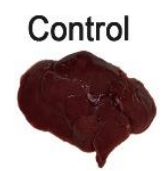

b

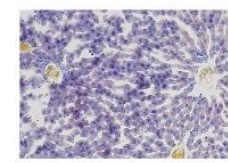

c

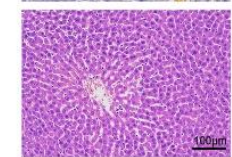

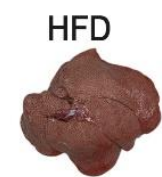
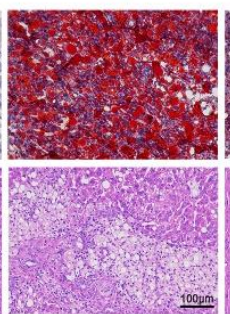
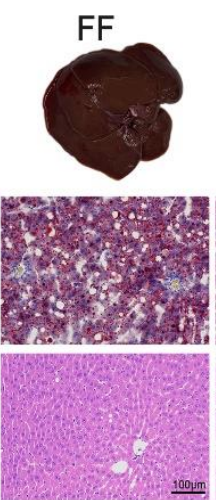
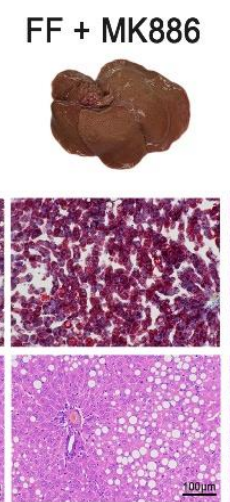
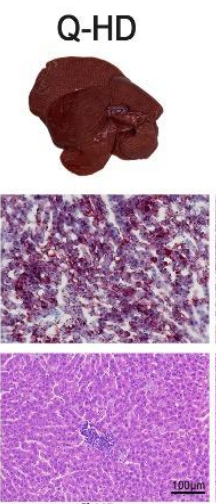
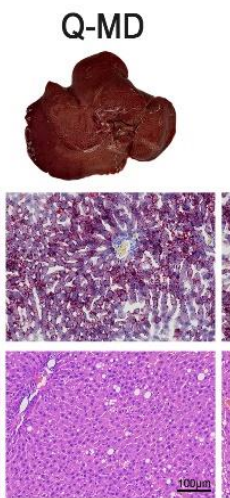
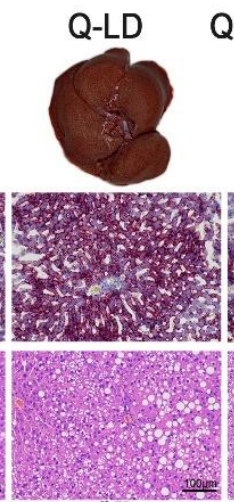
expression was markedly reduced in the two MK886-treated groups. Simultaneously, the expression of PPAR $\alpha$, CPT1- 
$\mathrm{L}, \mathrm{CPT} 2, \mathrm{MCAD}$ and LCAD was not apparent in the Control and HFD treatments. It is worth noting that the expression levels of CPT1-L and CPT2 in the Q-HD + MK886 group were lower than those in FF + MK886. Furthermore, the expression levels of MCAD and LCAD in the Q-HD + MK886 group were higher than those in FF + MK886.

183 184 185

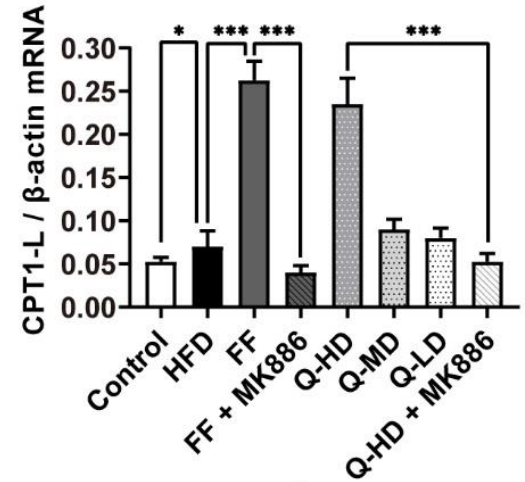

a

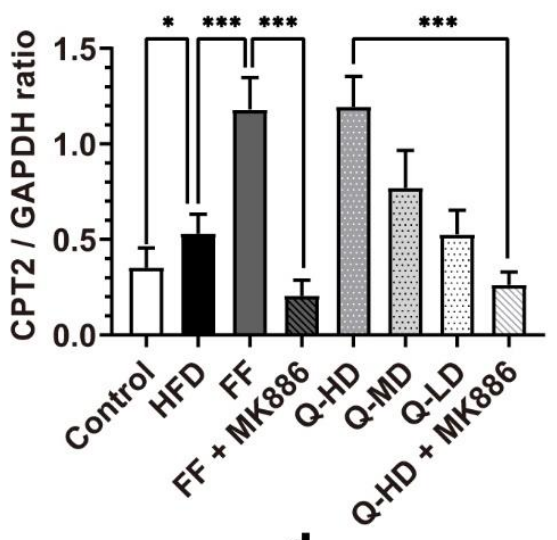

d

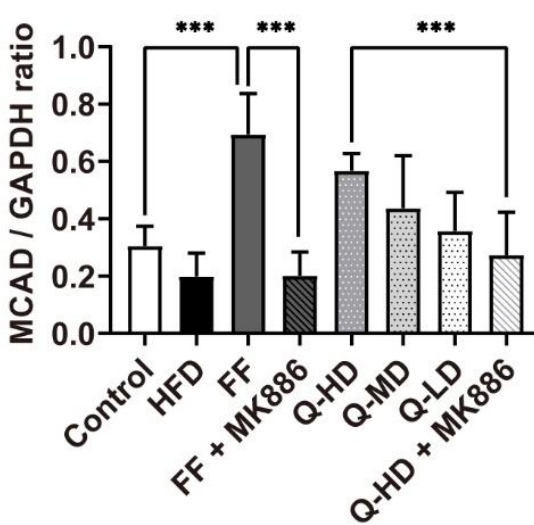

g

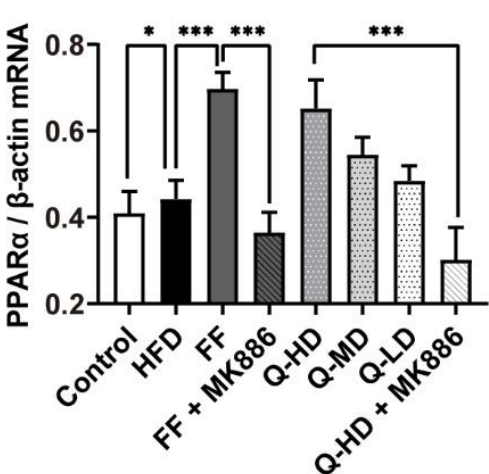

b
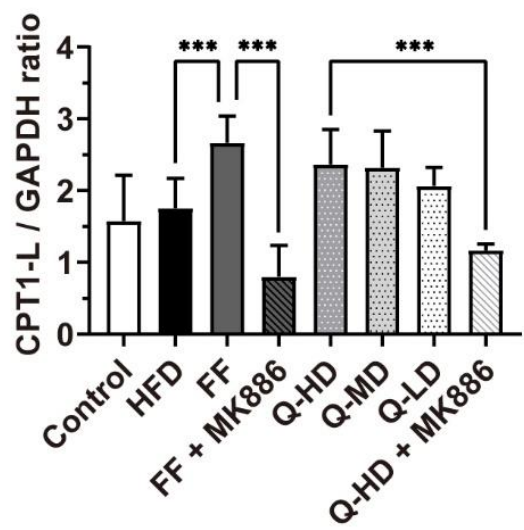

e

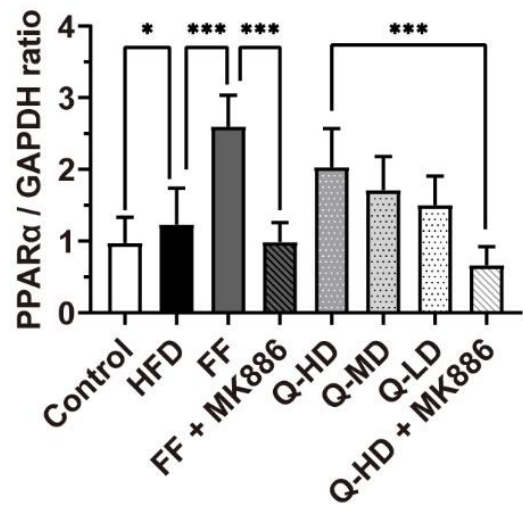

h

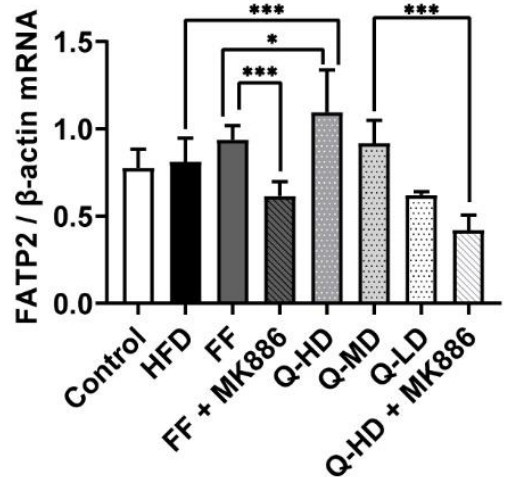

C
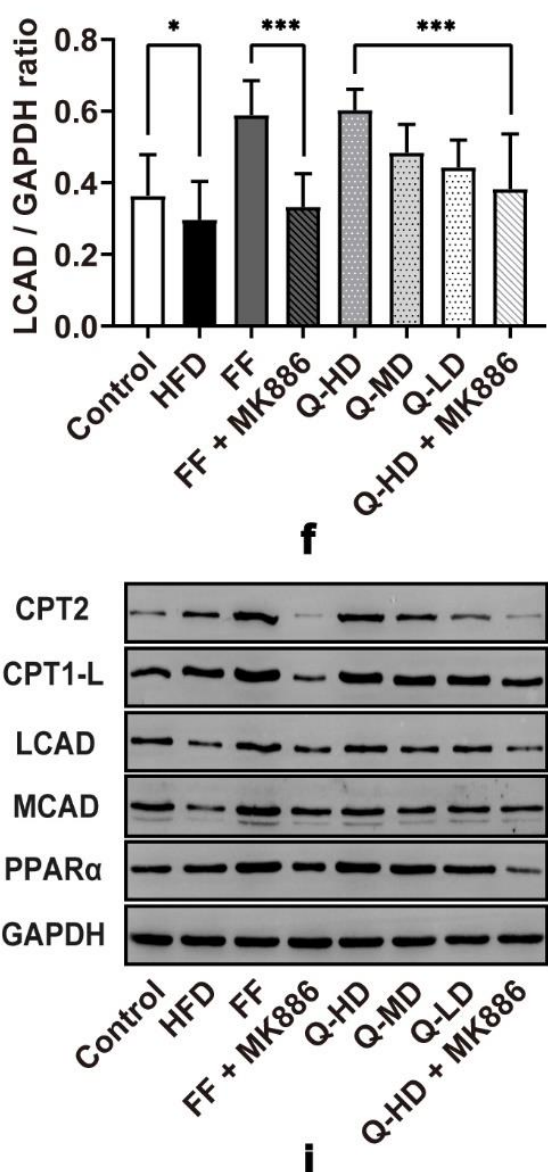

Figure $5(\mathrm{a}-\mathrm{c})$ Quercetin up-regulated the expression of CPT1-L, PPAR $\alpha$, and FATP2 mRNA based on a quantitative real-time polymerase chain reaction analysis. GAPDH was used as one invariant control. (d-i) Representative immunoblots of CPT2, CPT1-L, LCAD, MCAD and PPAR $\alpha$ in rat livers are indicated by western blot analysis. Relative expression levels were normalized to $\beta$-actin levels. All statistical data were expressed as the mean $\pm \mathrm{SD}, \mathrm{n}=10 . * \mathrm{P}<0.05, * * * \mathrm{P}<0.01$

\section{Discussion}

In the present experimental verification study, a stable NAFLD rat model was established. We confirmed that ORO staining, serum levels of TC and TG, and epididymal fat weights were more accurately alleviated in quercetin groups than HFD group, improving the synthesis and storage of triglycerides in the liver and body. In addition, HE staining, serum biochemical markers and hepatic inflammatory factors were ameliorated by quercetin further demonstrating the beneficial effects on liver cell damage and hepatic fibrosis. To investigate the presumed mechanisms underlying the regulation of fatty acid oxidation by network pharmacology, RT-qPCR and western blotting were used to investigate 
the protein expression levels. We observed an increasing trend in the mRNA and protein expression of PPAR $\alpha$, CPT1$\mathrm{L}, \mathrm{CPT} 2$, FATP2, MCAD and LCAD in the quercetin group.

Although previous studies demonstrated the potential therapeutic role of quercetin in fatty liver diseases 15,16 , a further comprehensive evaluation of quercetin's bioactivities, the identification of specific molecular targets, and structure-activity relationships are urgently needed. In this study, it seemed credible to verify the fatty acid oxidation pathway of quercetin in NAFLD using network pharmacology, and our western blotting results also revealed that other signaling pathways would affect corresponding proteins more than the PPAR pathway. In addition, our enrichment analyses have identified other pathways that potentially interact with Coptidis Rhizoma and Evodiae Fructus. Further experimental evidence is required to explore the mechanisms, and it remains to be investigated whether they are also involved in aberrant hepatic fatty acid metabolism in the liver.

NAFLD is a chronic progressive liver disease with a continuum of harmful conditions that disturb metabolic processes ${ }^{17}$. As an excessive supply of nutrients causes NAFLD, promising lifestyle modifications, such as weight loss through exercise can alleviate NAFLD. However, it remains challenging to achieve and sustain such modifications for most patients, and a substantial proportion of them are dramatically lean ${ }^{18,19}$. Quercetin is a well-known flavonoid isolated from the Chinese herb Coptidis Rhizoma and Evodiae Fructus, has a wide range of pharmacological properties ${ }^{20}$. Many studies have demonstrated quercetin's efficacy as a hypolipidemic and NAFLD protectant in animal experiments and clinical trials, making it a candidate for future pharmacological therapy against NAFLD 16,21-23. Additionally, liver inflammation and other pathophysiological processes, such as oxidative stress and energy consumption, are also targets of quercetin in NAFLD treatment ${ }^{24,25}$.

FAO is the major catabolic process for the degradation of long-chain fatty acids and plays a significant role in NAFLD's progression ${ }^{26}$. FAO is related with a series of enzymes, transporters, and other facilitating proteins ${ }^{27}$, such as PPAR $\alpha$, MCAD and LCAD which carry out the fatty acid cellular uptake, mitochondria shuttle, and the $\beta$-oxidation steps ${ }^{28,29}$.

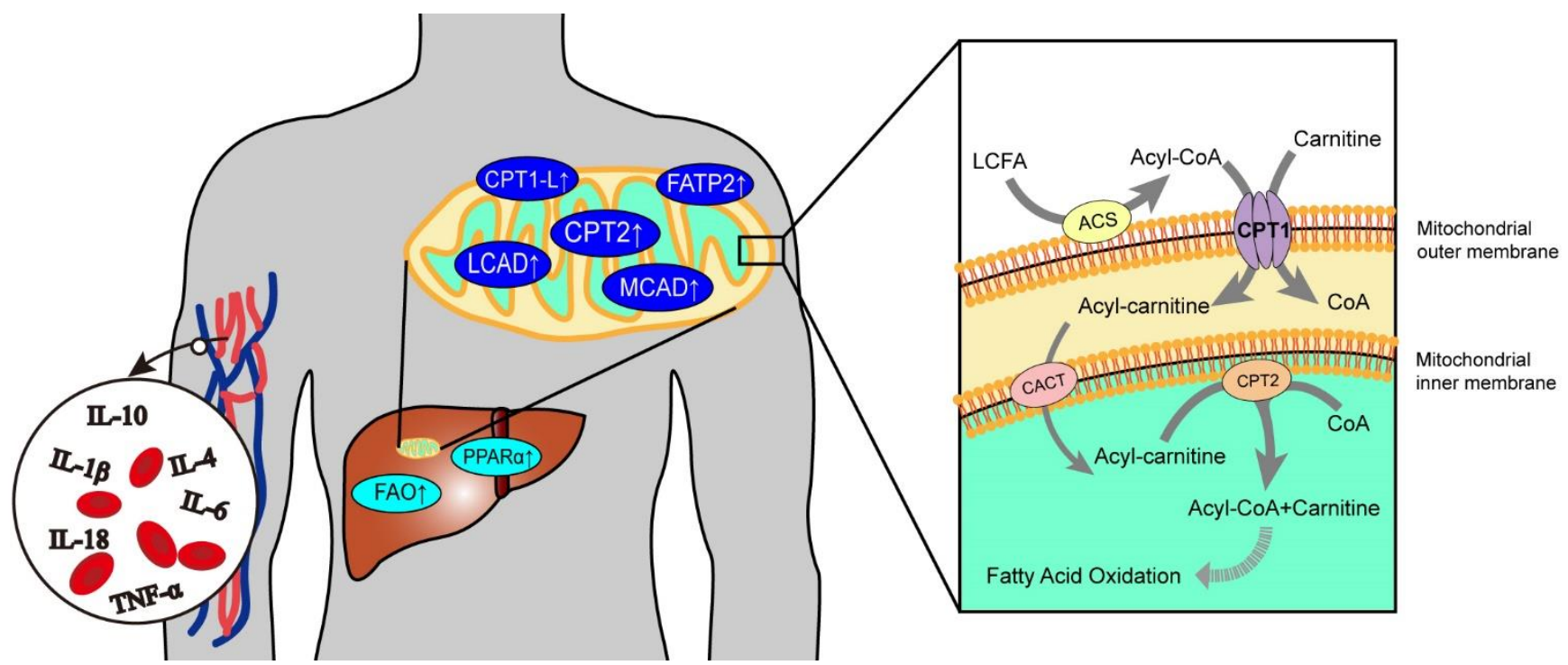

Figure 6 Carnitine O-palmitoyltransferase 1 is anchored on the mitochondrial outer membranes and takes charge of converting acylCoAs into acylcarnitines that are shuttled across the mitochondria membranes by the translocase and converted back to acyl-CoAs by carnitine O-palmitoyltransferase 2 inside mitochondria before undergoing fatty acid oxidation. Increasing the expression of PPAR $\alpha$, CPT1-L, CPT2, MCAD, LCAD and FATP2 enhances the capacity of hepatocytes to mediate $\beta$-oxidation of fatty acids and minimizes lipid accumulation and inflammation response, which results in NAFLD.

PPAR $\alpha$ is a nuclear receptor highly expressed in hepatocytes and is involved in fatty acid synthesis, transport, and storage ${ }^{30-32}$. The reduction of hepatic PPAR $\alpha$ expression causes transcriptional impairment of related target genes ${ }^{33}$. FATP2 activates long-chain fatty acids (LCFAs) as a very long-chain acyl-CoA synthetase and to transport LCFAs as a fatty acid transporter ${ }^{34}$. CPT1-L converts acyl-CoAs into acylcarnitines passing through mitochondrial membranes by major translocases as an essential rate-limiting enzyme of the outer mitochondrial membrane and is transformed into acyl-CoAs by CPT2 before undergoing $\beta$-oxidation ${ }^{35}$. Primarily, the pathways described above are closely related to the pathogenesis of NAFLD. (Figure 6) 
With the help of the TCMSP, all active ingredients contained in Coptidis Rhizoma and Evodiae Fructus were obtained. The TCMSP database also includes pharmacokinetic properties for natural compounds involving oral bioavailability $(\mathrm{OB})$, drug-likeness (DL) and etc. Drugs are absorbed by the gastrointestinal tract and pass through the liver to enter the systemic circulation, and the $\mathrm{OB}$ is the absorption percentage of the orally administered drug. Meanwhile, to reduce the risks inherent to drug design and development, the DL properties are used to evaluate the potential failure characteristics of compounds.

In this study, the chemical constituents in the compounds of Coptidis Rhizoma and Evodiae Fructus that had OB $\geqslant$ $30 \%$ and DL $\geqslant 0.18$ were chosen as the active ingredients and then gene or protein targets linked to selected compounds were also retrieved from the TCMSP. Conversely, DisGeNET database was employed to identify NAFLD-related genes as expected. Furthermore, all standard gene names and UniProt IDs of the target proteins were manually obtained from the UniProt Knowledgebase.

\subsection{Network construction}

A network diagram intersected drug target genes and NAFLD-related genes, and the complex PPIs of each target were uploaded onto the PPI Network Functional Enrichment Analysis (STRING, https://string-db.org/), that provides physical and functional associations between numerous proteins. For the sake of showing these processes, Cytoscape v3.8.0 software was employed to perform a visual analysis of the drug-compound-gene-disease network. The tools served as media for converting datasets into classifications and platforms facilitating research and forecasting.

\subsection{Enrichment analyses}

GO terms and KEGG pathway enrichment analyses were conducted using the Database for Annotation, Visualization, and Integrated Discovery (DAVID) v6.8 (https://david.ncifcrf.gov/tools.jsp) to understand the enriched biological meaning behind differentially expressed underlying genes and proteins. GO enrichment includes cell component (CC, cell component), molecular function (MF, molecular function), and biological process (BP, biological process). The KEGG pathway represents molecular interactions, reactions, and relation networks among other systems. Ultimately, the core targets such as PPAR $\alpha$, MCAD, LCAD, CPT1-L, CPT2 and FATP2 which participate in FAO signaling pathway were selected to perform further in vivo experiments.

\subsection{Molecular docking analysis}

Molecular docking analysis provided a visual explanation of the interaction between quercetin and its potential protein targets associated with NAFLD. The three-dimensional (3D) structures of receptor proteins were downloaded from the RCSB PDB database (https://www.rcsb.org/). The two-dimensional (2D) structure of quercetin was downloaded from the PubChem database (https://pubchem.ncbi.nlm.nih.gov/) and transformed into PDB format as the molecule ligand. Chem3D 17.0 was used to export 3D chemical structures and minimize their energy. PyMOL 2.4.0 software was performed the dehydration of receptor proteins and AutoDock 4.2 software was used to carry out hydrogenation and charge calculation of proteins. Finally, AutoDock Vina 1.1.2 was used to dock small molecule ligands of quercetin with target receptor proteins.

\subsection{Experimental animals}

A total of 96 healthy male Sprague Dawley rats (body weight 200-220 g) were obtained from Hunan SJA Laboratory Animal Co., Ltd., Changsha, Hunan, China (SCXK 2019-0004). All experimental rats had access to food and water ad libitum and were housed in an environmentally controlled room (temperature $24 \pm 2{ }^{\circ} \mathrm{C}$, humidity $55 \% \pm 5 \%$, with a 12-h light-dark cycle). All experimental rats were weighed twice a week, housed five per cage under controlled conditions, and fed with basic forage for one week before the experiments for acclimatization. All animal procedures were carried out in compliance with the ARRIVE guidelines and use of laboratory animals established by the Institutional Animal Care and Use Committee of Chengdu University of Traditional Chinese Medicine (TCM 2016-312).

\subsection{Experimental design and supplementation}

Sprague Dawley rats $(n=96)$ were randomly distributed into two groups: NAFLD model group and the Control group. The Control group $(n=12)$ was fed with basic forage, while 84 rats were fed a high-fat diet (HFD) for 10 weeks to induce NAFLD. The HFD contained basic forage supplemented with $0.2 \%$ propylthiouracil, $1 \%$ cholesterol, $1 \%$ sodium tauroglycocholate, 5\% yolk powder, and 10\% lard (Ensiweier Biotech Co., Ltd., Chengdu, Sichuan, China).

Subsequently, NAFLD rats were subdivided into 7 groups $(\mathrm{n}=12$ rats/group) by systematic sampling: HFD, Fenofibrate (FF, 100 mg/kg/day b.w.), FF + MK886 (FF, 100 mg/kg/day b.w., MK886, $1 \mathrm{mg} / \mathrm{kg} /$ day b.w.), quercetin at a high dose (Q-HD, $100 \mathrm{mg} / \mathrm{kg} /$ day b.w.), quercetin at a medium dose (Q-MD, $50 \mathrm{mg} / \mathrm{kg} /$ day b.w.), quercetin at a low dose (QLD, 25 mg/kg/day b.w.), Q-HD + MK886 (Q-HD, 100 mg/kg/day b.w., MK886, 1 mg/kg/day b.w.).

Fenofibrate, a synthetic agonist of PPAR $\alpha$, was used as a positive control. MK886, a non-competitive PPAR $\alpha$ antagonist, provided a reliable scientific basis for this study. In detail, quercetin and fenofibrate were separately dissolved 
in $0.5 \%$ sodium carboxymethyl cellulose, and MK886 was dissolved in $20 \%$ dimethyl sulfoxide. These compounds were administered to rats by gavage at a dose of $10 \mathrm{~mL} / \mathrm{kg}$ body weight for 4 consecutive weeks with HFD. Likewise, the control group $(n=12)$ was fed with a basic forage diet. Both the HFD and Control groups were treated with $0.5 \%$ sodium carboxymethyl cellulose as controls each day from the beginning of pharmacological intervention. All animals were fasted for $24 \mathrm{~h}$ before they were sacrificed, and all surgeries were performed under sodium pentobarbital anesthesia to minimize the experimental damage.

\subsection{Reagents}

Quercetin (purity $>98 \%$ ) were supplied by Sichuan Weikeqi Biotech Co., Ltd. (wkq20061112, Chengdu, Sichuan, China). The PPAR $\alpha$ antagonist MK886 was purchased from MedChemExpress USA (HY-14166/CS-5755, Monmouth Junction, NJ, USA). Fenofibrate were obtained from Affiliated Hospital of Chengdu University of Traditional Chinese Medicine (29681, Recipharm Fontaine, Rue des Pres Potets, Fontaine les Dijon, France). Dimethyl sulfoxide and sodium carboxymethyl cellulose were obtained from Sigma-Aldrich Co., Ltd. (St. Louis, MO, USA).

\subsection{Biochemical assays}

Assay kits for measuring TC, TG, ALT, AST, ALP, and $\gamma$-GT were purchased from Nanjing Jiancheng Bioengineering Institute (Nanjing, Jiangsu, China). Elisa kits for measuring TNF- $\alpha$, IL-6 and IL-1 $\beta$ in rat livers were obtained from Multi Sciences Biotech Co., Ltd. (Hangzhou, Zhejiang, China). Serum levels and hepatic inflammatory factor levels were measured according to the manufacturer's instructions.

\subsection{Liver histopathological analyses}

All rat liver tissues were fixed with formaldehyde after washing. Paraffin-embedded liver sections were sectioned at a $6 \mu \mathrm{m}$ thickness. After deparaffinization and hydration, liver tissues were stained with hematoxylin and eosin; the histopathological characteristics were observed under a high-resolution upright microscope with photographic capability.

\subsection{Lipid accumulation analyses}

To evaluate quercetin's effect on lipid accumulation, epididymal fat weight, liver morphology, liver index and hepatic ORO staining were performed. All rats were weighed, narcotized, sacrificed, and then the liver indices were calculated and observed. The optimal cutting temperature-embedded frozen liver tissues were cut into $10 \mu \mathrm{m}$ thick sections and fixed with $75 \%$ alcohol. The frozen liver sections were immersed in a $60 \%$ isopropanol solution and stained with ORO solution for $2 \mathrm{~min}$. The samples were then washed and counterstained with hematoxylin for 3 min. Photomicrographs were captured at a magnification of 200×. Images were analyzed using Image-Pro Plus 6.0 software (Media Cybernetics, Inc., Rockville, MD, USA), where the percentage of the ORO-positive area was calculated per image area to yield the relative staining level for each sample.

\subsection{RNA isolation and real-time quantitative PCR}

Total RNA from fresh liver samples was extracted using TRIzol Reagent (Thermo Scientific, Waltham, MA, USA) according to the manufacturer's protocol, and then $2.0 \mu \mathrm{g}$ RNA was reverse-transcribed into cDNA [5X All-In-One MasterMix (with AccuRT Genomic DNA Removal Kit)] (\#G492, Applied Biological Materials Inc, British Columbia, Canada). Real-time quantitative PCR was conducted using EvaGreen 2X qPCR MasterMix (0194844830001, Applied Biological Materials Inc). The PCR cycling profile was as follows: one cycle at $50^{\circ} \mathrm{C}$ for $2 \mathrm{~min}, 95^{\circ} \mathrm{C}$ for $10 \mathrm{~min}, 40$ cycles at $95^{\circ} \mathrm{C}$ for $15 \mathrm{~s}$, and $60^{\circ} \mathrm{C}$ for $60 \mathrm{~s}$. Relative mRNA expression was quantified using the $2^{-\Delta \Delta \mathrm{C}_{\mathrm{T}}}$ value. Each sample was amplified in triplicate, and $\beta$-actin expression was used as an internal control. The primers used were designed and validated by Sangon Biotech Co., Ltd. (Shanghai, China): $\beta$-actin, forward 5'-TGTCACCAACTGGGACGATA-3' and reverse 5'-GGGGTGTTGAAGGTCTCAAA-3'; $c p t 1 a$, forward 5'-TGCCAGCAAGCATACATCACC-3` and reverse 5'TGCCCAGACCTACCTATTGCTC-3’; Ppara, forward 5`- AGGATGGCAGGAGCAGGTAGATG-3` and reverse 5`TGTTGGCGATGGCGGTATTGC-3’; Fatp2 forward 5'-CAGCAAGCAAGCCAGAGACATCC-3` and reverse 5`-CCAGCATCCACATACAAGGCAGAC-3'.

\subsection{Western Blot of PPAR $\alpha, C P T 1-L, C P T 2, M C A D$, and LCAD}

Total liver protein was extracted from hepatic tissues using RIPA lysis buffer (Multi Sciences Biotech Co., Ltd.). The BCA Protein Assay Kit (Multi Sciences Biotech Co., Ltd.) was used for protein concentration measurements. The protein samples $(50 \mu \mathrm{g})$ were separated on 10\% SDS-PAGE and transferred onto 0.22 polyvinylidene fluoride membranes (Millipore, Billerica, MA, USA). The primary antibodies used in this study were GAPDH (Mab5465-100, Multi Sciences Biotech Co., Ltd., 1:2,000), PPAR $\alpha$ (b3501, YT3835, ImmunoWay Biotechnology, Newark, DE, USA, 1:1000), CPT1-L (GR3351270-2, ab234111, Abcam, Cambridge, UK, 1:1,000), CPT2 (HN0927, ET1611-64, HUABIO, China, 1:1,000), LCAD (GR82379-5, ab234111, Abcam), and MCAD (YT5024, ImmunoWay Biotechnology). Membranes were incubated with secondary antibodies (goat anti-rabbit, 1:10,000 dilution) for $80 \mathrm{~min}$ at $37^{\circ} \mathrm{C}$, then the antibody-bound 
proteins were detected via enhanced chemiluminescence. Lastly, the immunoblots were visualized using a ChemiScope 6100 (Shanghai Qinxiang Scientific Instrument Co., Ltd., Shanghai, China).

\subsection{Statistical analyses}

The data from in vivo experiments were expressed as the mean \pm standard deviation (SD). The differences between two groups were used by Student's t-test. The multiple comparisons were determined using one-way analysis of variance followed by the Tukey post hoc test, with $\mathrm{P}<0.05$, considered statistically significant. All experiments were repeated at least three times.

\section{Conclusions}

In conclusion, quercetin reduced hepatic lipid accumulation and inflammation, ameliorated pathological liver changes, and up-regulated the expression levels of hepatic effectors in fatty acid oxidation. The results suggest the potential of quercetin as a nutritional supplement to HFD-induced NAFLD. Furthermore, the antagonist and agonist of PPAR $\alpha$ provided a reliable scientific basis that quercetin ameliorated NAFLD by activating the PPAR pathway. Combining system network pharmacology strategy and molecular docking technology with in vivo experimental study may prove to be a useful tool for evaluating bioactive ingredients for NAFLD.

\section{Additional Information}

Competing interests: The authors declare no competing interests.

Funding: This research was supported by the grant from the National Natural Science Foundation of China, grant number 81973743.

Ethics approval and consent to participate: All animal procedures were conformed to the guidelines for the care and use of laboratory animals established by the Institutional Animal Care and Use Committee of Chengdu University of Traditional Chinese Medicine (TCM 2016-312).

The study was carried out in compliance with the ARRIVE guidelines.

Informed Consent Statement: Not applicable.

\section{References}

1 Younossi, Z. et al. Global burden of NAFLD and NASH: trends, predictions, risk factors and prevention. Nat Rev Gastroenterol Hepatol 15, 11-20, doi:10.1038/nrgastro.2017.109 (2018).

2 Friedman, S. L., Neuschwander-Tetri, B. A., Rinella, M. \& Sanyal, A. J. Mechanisms of NAFLD development and therapeutic strategies. Nat Med 24, 908-922, doi:10.1038/s41591-018-0104-9 (2018).

Reimer, K. C., Wree, A., Roderburg, C. \& Tacke, F. New drugs for NAFLD: lessons from basic models to the clinic. Hepatol Int 14, 8-23, doi:10.1007/s12072-019-10001-4 (2020).

Moore, J. B. Non-alcoholic fatty liver disease: the hepatic consequence of obesity and the metabolic syndrome. Proc Nutr Soc 69, 211-220, doi:10.1017/S0029665110000030 (2010). 1257, doi:10.1016/j.jhep.2016.07.033 (2016).

Fritzen, A. M., Lundsgaard, A.-M. \& Kiens, B. Tuning fatty acid oxidation in skeletal muscle with dietary fat and exercise. Nat Rev Endocrinol 16, 683-696, doi:10.1038/s41574-020-0405-1 (2020). (2017). Insulin Resistance. Endocr Rev 40, 1367-1393, doi:10.1210/er.2019-00034 (2019). 

doi:10.1016/j.apsb.2019.11.017 (2020).

Rosell, M. \& Fernández-Recio, J. Docking approaches for modeling multi-molecular assemblies. Curr Opin Struct Biol 64, 59-65, doi:10.1016/j.sbi.2020.05.016 (2020).

Wang, X., Wang, Z.-Y., Zheng, J.-H. \& Li, S. TCM network pharmacology: A new trend towards combining computational, experimental and clinical approaches. Chin J Nat Med 19, doi:10.1016/S1875-5364(21)60001-8 (2021). $\mathrm{Ru}$, J. et al. TCMSP: a database of systems pharmacology for drug discovery from herbal medicines. J Cheminform 6, 13, doi:10.1186/1758-2946-6-13 (2014).

Gori, M. et al. Quercetin and hydroxytyrosol as modulators of hepatic steatosis: A NAFLD-on-a-chip study. Biotechnol Bioeng 118, 142-152, doi:10.1002/bit.27557 (2021).

Kobori, M., Akimoto, Y., Takahashi, Y. \& Kimura, T. Combined Effect of Quercetin and Fish Oil on Oxidative Stress in the Liver of Mice Fed a Western-Style Diet. J Agric Food Chem 68, 13267-13275, doi:10.1021/acs.jafc.0c02984 (2020).

Bessone, F., Razori, M. V. \& Roma, M. G. Molecular pathways of nonalcoholic fatty liver disease development and progression. Cell Mol Life Sci 76, 99-128, doi:10.1007/s00018-018-2947-0 (2019).

Rinella, M. E. \& Sanyal, A. J. Management of NAFLD: a stage-based approach. Nature reviews. Gastroenterology $\mathcal{E}$ hepatology 13, 196-205, doi:10.1038/nrgastro.2016.3 (2016).

Wang, M., Ma, L.-J., Yang, Y., Xiao, Z. \& Wan, J.-B. n-3 Polyunsaturated fatty acids for the management of alcoholic liver disease: A critical review. Crit Rev Food Sci Nutr 59, S116-S129, doi:10.1080/10408398.2018.1544542 (2019).

Porras, D. et al. Protective effect of quercetin on high-fat diet-induced non-alcoholic fatty liver disease in mice is mediated by modulating intestinal microbiota imbalance and related gut-liver axis activation. Free Radic Biol Med 102, 188-202, doi:10.1016/j.freeradbiomed.2016.11.037 (2017).

Brüll, V. et al. No effects of quercetin from onion skin extract on serum leptin and adiponectin concentrations in overweight-to-obese patients with (pre-)hypertension: a randomized double-blinded, placebo-controlled crossover trial. Eur J Nutr 56, 2265-2275, doi:10.1007/s00394-016-1267-0 (2017). Eid, H. M. \& Haddad, P. S. The Antidiabetic Potential of Quercetin: Underlying Mechanisms. Curr Med Chem 24, 355-364, doi:10.2174/0929867323666160909153707 (2017).

Li, Y. et al. Quercetin, Inflammation and Immunity. Nutrients 8, 167, doi:10.3390/nu8030167 (2016).

Lin, R., Piao, M., Song, Y. \& Liu, C. Quercetin Suppresses AOM/DSS-Induced Colon Carcinogenesis through Its AntiInflammation Effects in Mice. J Immunol Res 2020, 9242601, doi:10.1155/2020/9242601 (2020).

Vanani, A. R., Mahdavinia, M., Shirani, M., Alizadeh, S. \& Dehghani, M. A. Protective effects of quercetin against oxidative stress induced by bisphenol-A in rat cardiac mitochondria. Environ Sci Pollut Res Int 27, 15093-15102, doi:10.1007/s11356-020-08048-0 (2020).

Xiong, J. Fatty Acid Oxidation in Cell Fate Determination. Trends Biochem Sci 43, 854-857, doi:10.1016/j.tibs.2018.04.006 (2018).

Zhang, Y., Bharathi, S. S., Beck, M. E. \& Goetzman, E. S. The fatty acid oxidation enzyme long-chain acyl-CoA dehydrogenase can be a source of mitochondrial hydrogen peroxide. Redox Biol 26, 101253, doi:10.1016/j.redox.2019.101253 (2019). steatosis in sirtuin-5 knockout mice. Sci Rep 10, 18367, doi:10.1038/s41598-020-75615-3 (2020). disease 18, 6, doi:10.1186/s12944-018-0933-z (2019).

Brocker, C. N. et al. Extrahepatic PPAR $\alpha$ modulates fatty acid oxidation and attenuates fasting-induced hepatosteatosis in mice. J Lipid Res 59, 2140-2152, doi:10.1194/jlr.M088419 (2018). 
31 Kersten, S. Integrated physiology and systems biology of PPARalpha. Mol Metab 3, 354-371, doi:10.1016/j.molmet.2014.02.002 (2014). Montagner, A. et al. Liver PPARalpha is crucial for whole-body fatty acid homeostasis and is protective against NAFLD. Gut 65, 1202-1214, doi:10.1136/gutjnl-2015-310798 (2016). Westerouen Van Meeteren, M. J., Drenth, J. P. H. \& Tjwa, E. Elafibranor: a potential drug for the treatment of nonalcoholic steatohepatitis (NASH). Expert Opin Investig Drugs 29, 117-123, doi:10.1080/13543784.2020.1668375 (2020). Perez, V. M. et al. Deletion of fatty acid transport protein 2 (FATP2) in the mouse liver changes the metabolic landscape by increasing the expression of PPAR $\alpha$-regulated genes. J Biol Chem 295, 5737-5750, doi:10.1074/jbc.RA120.012730 (2020).

Author Contributions: T.S. and W.Z designed the work, J.L. made contributions to the conception, W.Z, D.Y. and X.R. performed the experiments, Y.C. performed the interpretation of data, J.W. contributed thoughtful comments on the project, W.Z and J.Z. wrote the manuscript; all authors have read and approved the final manuscript. 


\section{Figures}<smiles>O=c1c(O)c(-c2ccc(O)c(O)c2)oc2cc(O)cc(O)c12</smiles>

a

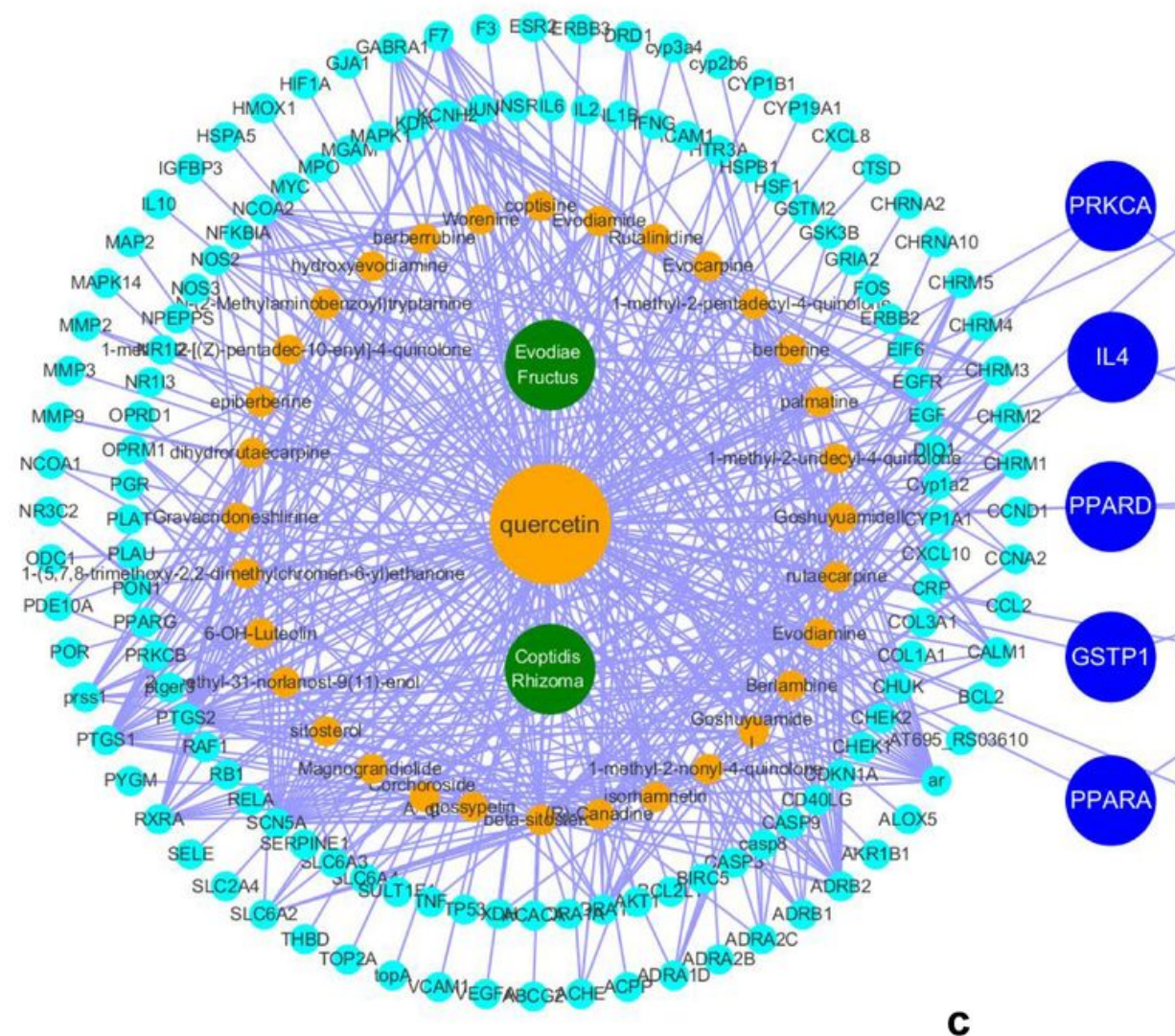

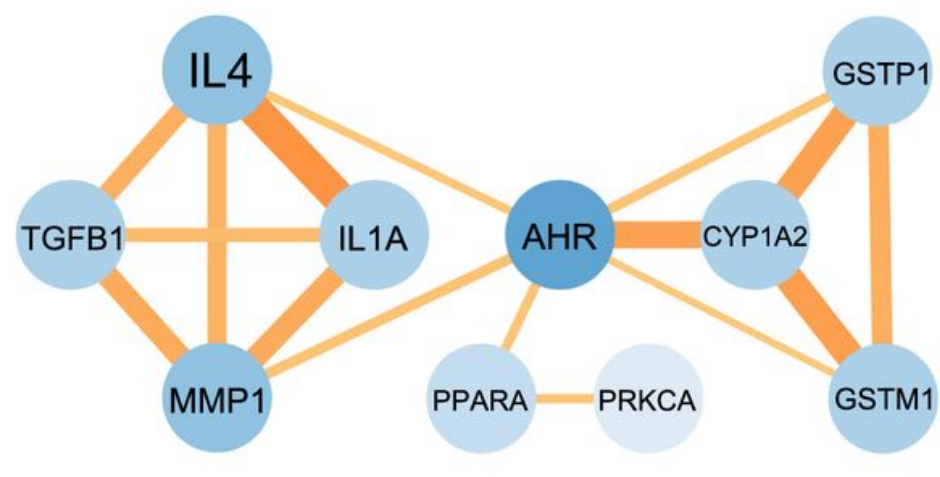

b
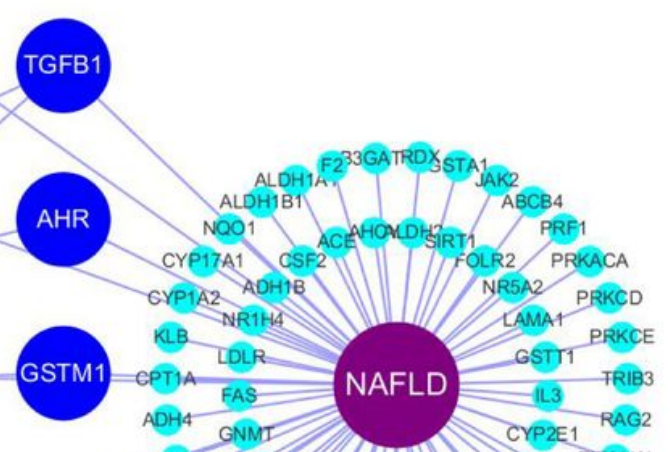

\section{Figure 1}

(a) The two-dimensional (2D) structure of quercetin. (b) The protein-protein interaction (PPI) network of proteins targeted by Coptidis Rhizoma and Evodiae Fructus and encoded by proteins associated with non-alcoholic fatty liver disease. (c) The potential drug-compound-gene-disease network. 


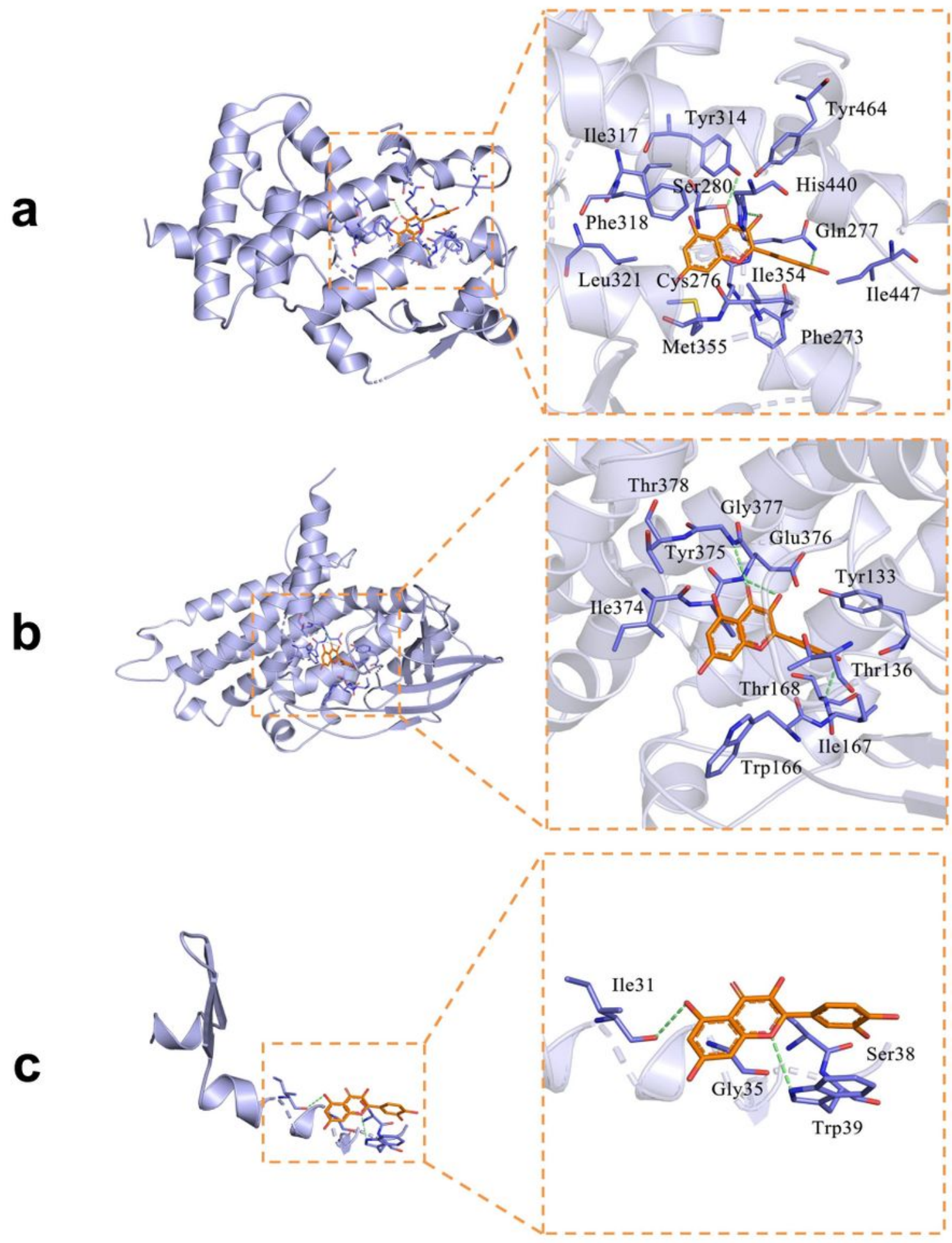

\section{Figure 2}

Molecular docking between the small molecule ligands of quercetin and 2REW (encoded by PPARa), 4P13 (encoded by MCAD), and 2LE3 (encoded by CPT1-L) protein receptors. 


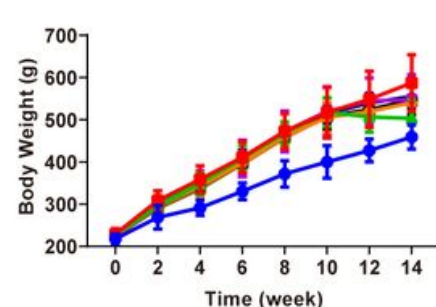

- Control $=\mathrm{HFD} \neq \mathrm{FF}+\mathrm{FF}+\mathrm{MK} 886$

$\leftarrow$ Q-HD $\bullet$ Q-MD $=$ Q-LD $\neq$ Q-HD + MK886

a
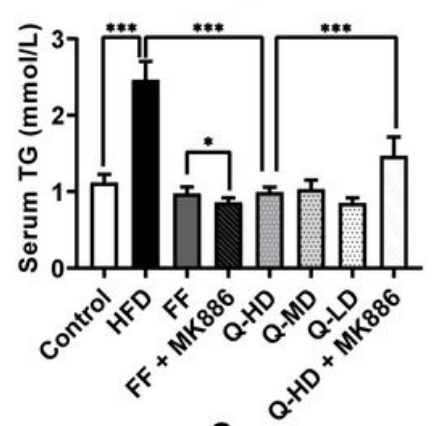

e
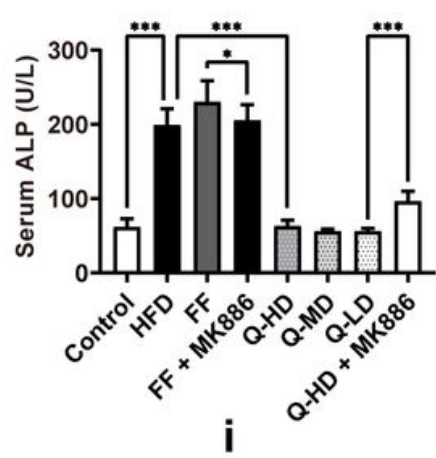

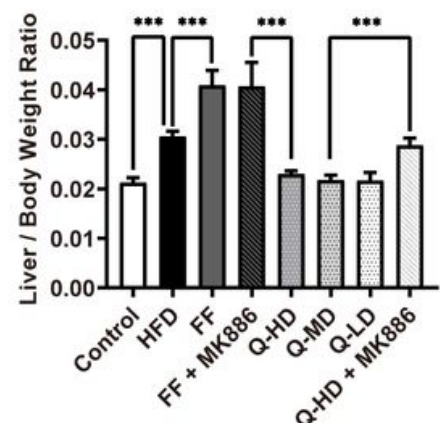

b
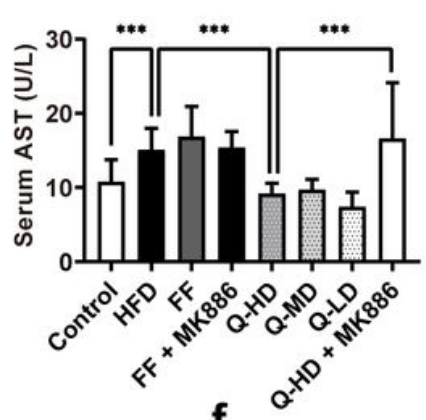

f

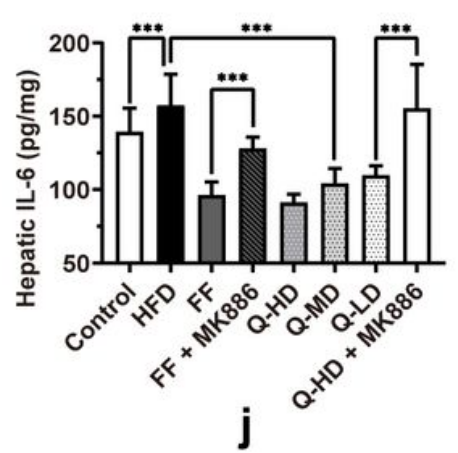

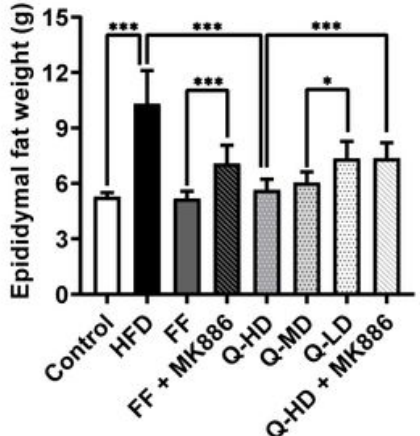

C
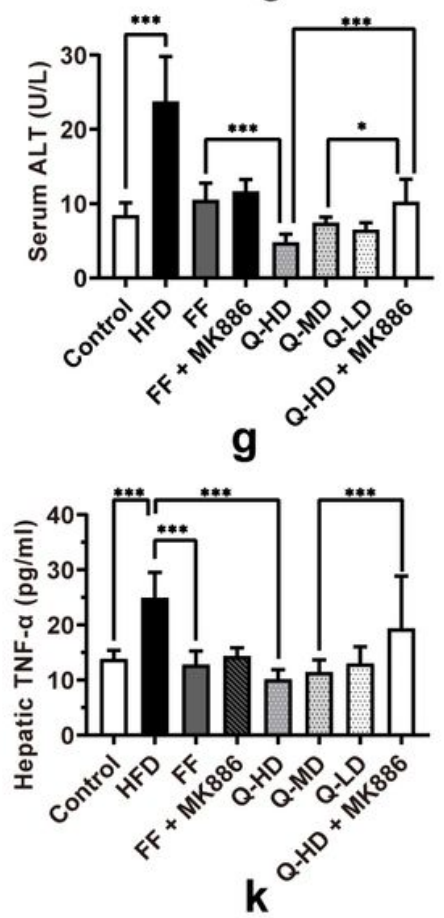
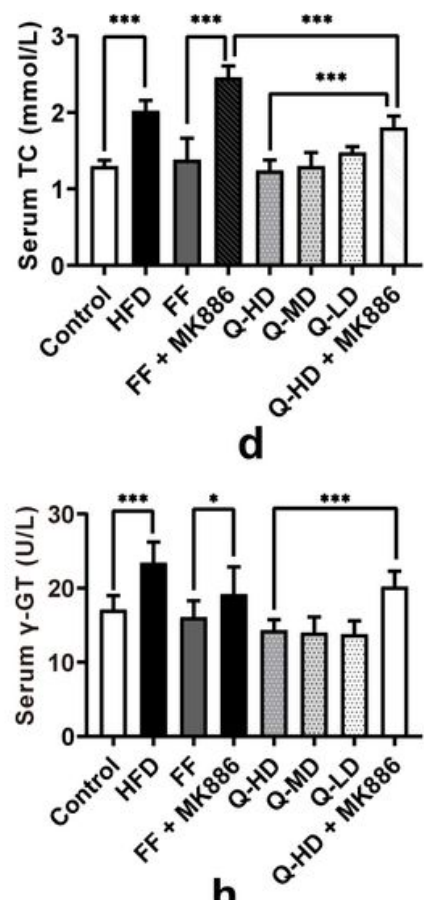

h

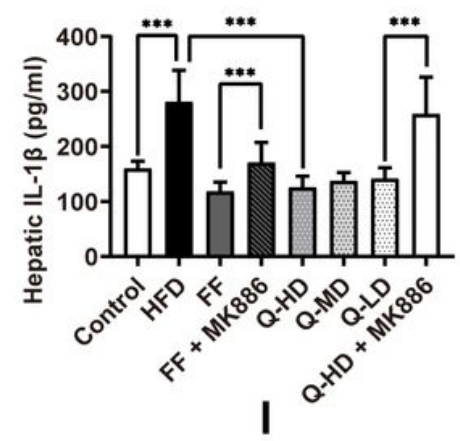

Figure 3

(a) Changes of body weight in different groups of rats. (b) The liver and body weight ratios (the liver indices) in different groups of rats. (c) The epididymal fat weights in different groups of rats. Two sensitive indices, the total cholesterol (d) and triglyc-erides (e), for quercetin effects on the lipid accumulation in the liver induced by a high-fat diet contents in liver tissue. The serum levels of aspartate aminotransferase $(\mathrm{f})$, alanine aminotransferase $(\mathrm{g})$, $\mathrm{y}$-glutamyl transferase $(\mathrm{h})$ and alkaline phosphatase (i) in ex-perimental groups. (j-I) Hepatic inflammatory factors levels of the tumor necrosis factor-a (TNFa), interleukin-6 (IL-6) and interleu-kin-1 $\beta$ (IL-1 $\beta$ ) were compared among all groups. All results were expressed as the mean $\pm S D, n=10$. $\otimes P<0.05$, $\triangle \otimes \otimes P<0.01$. 
a

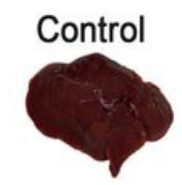

b

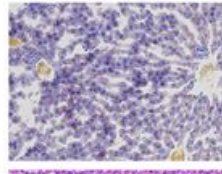

c

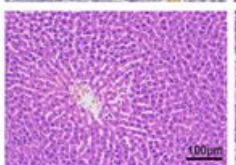

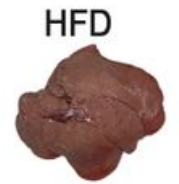
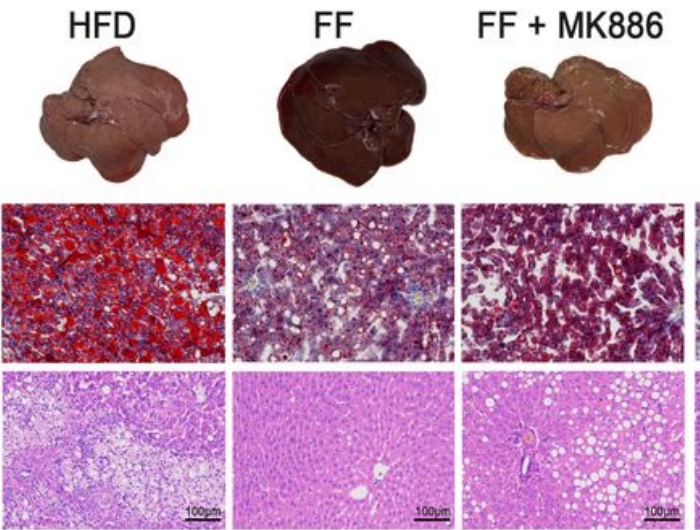
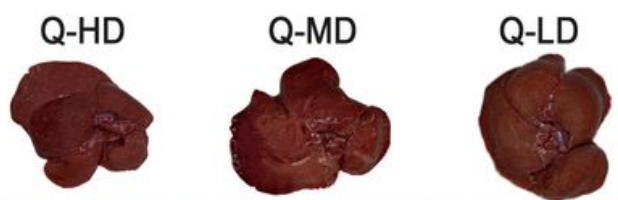

Q-HD + MK886
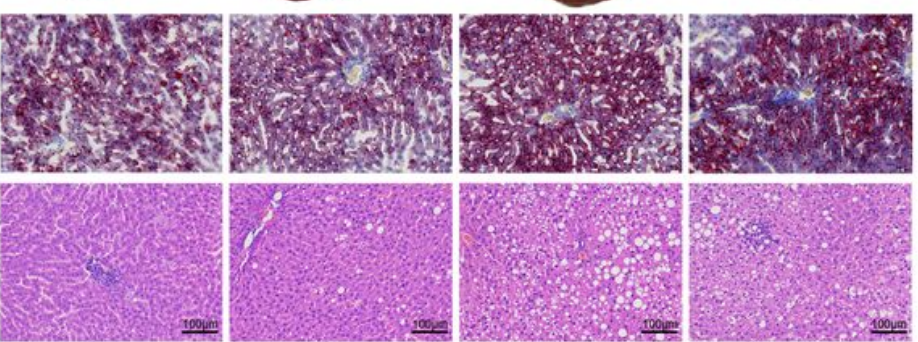

Figure 4

(a) The representative images from the morphology of the livers in all groups. Quercetin relieved fatty liver upon visual inspection. (b) The accumulation of lipids in liver samples from all groups expressed by Oil red 0 staining. (magnification, 200x). (c) The histopathology of hepatic tissues in different groups of rats as indicated by hematoxylin and eosin (HE) staining (magnification 200x). 


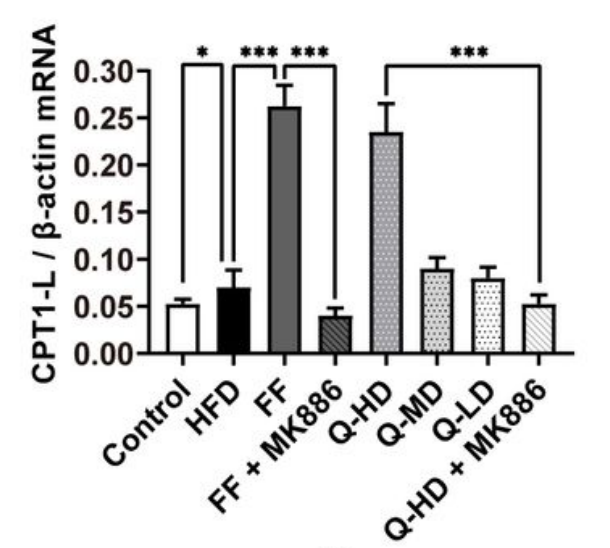

a
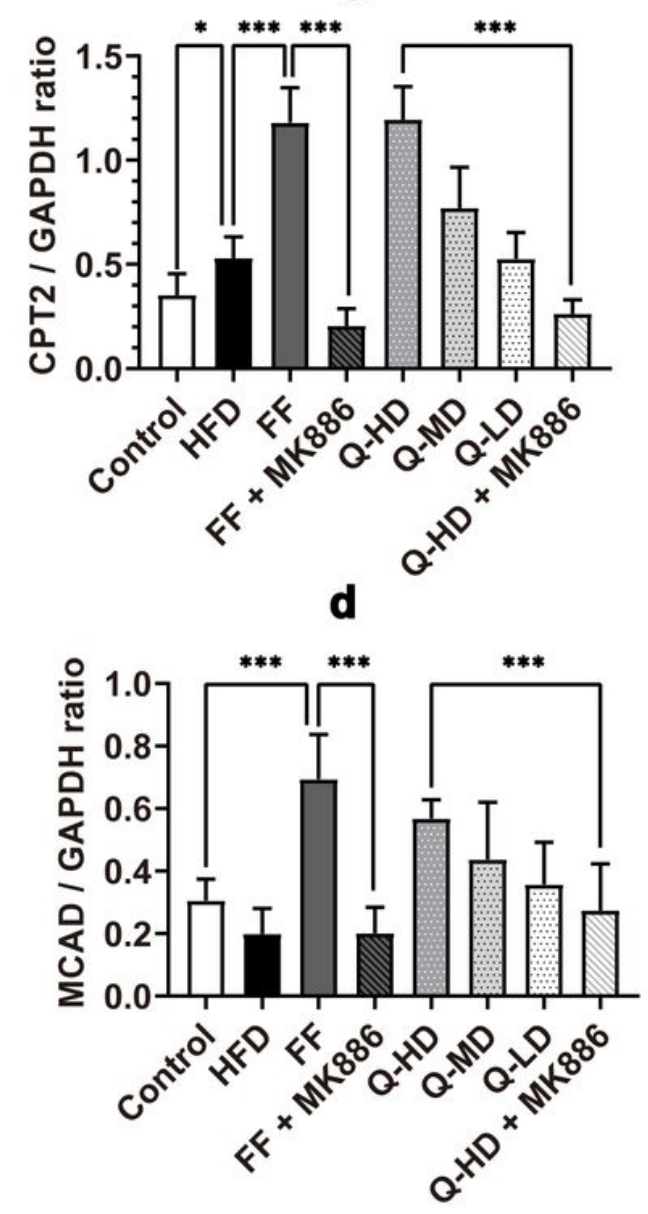

$\mathbf{g}$

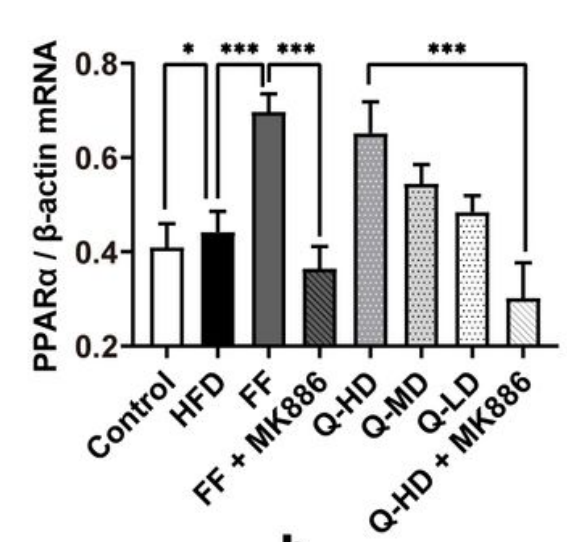

b
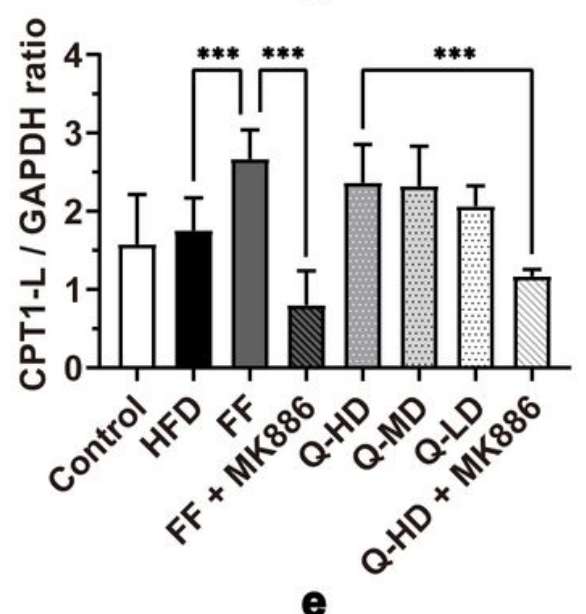

e

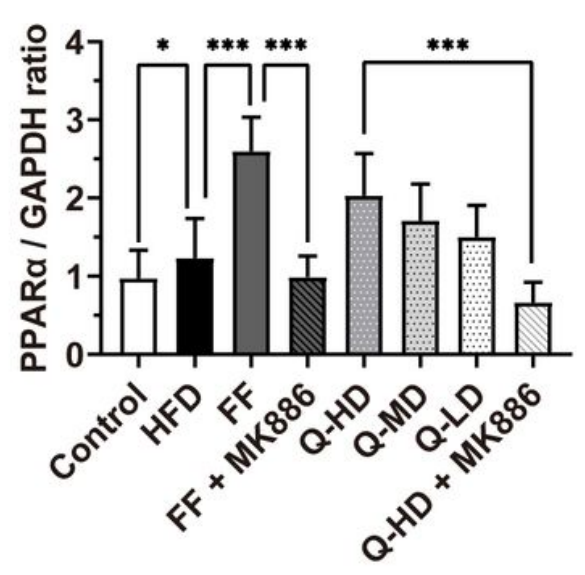

h

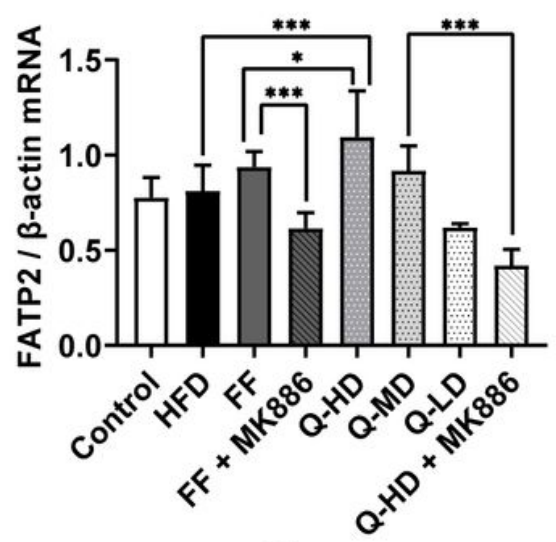

C
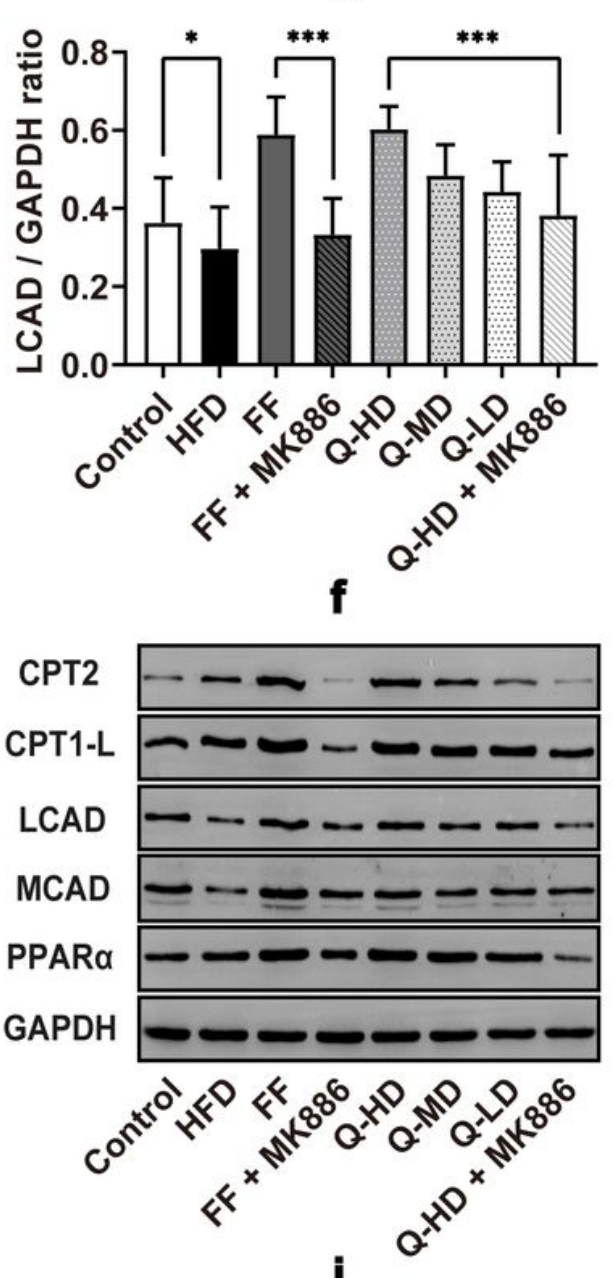

Figure 5

$(a-c)$ Quercetin up-regulated the expression of CPT1-L, PPARa, and FATP2 mRNA based on a quantitative real-time poly-merase chain reaction analysis. GAPDH was used as one invariant control. $(\mathrm{d}-\mathrm{i})$ Representative immunoblots of CPT2, CPT1-L, LCAD, MCAD and PPARa in rat livers are indicated by western blot analysis. Relative expression levels were normalized to $\beta$-actin levels. All statistical data were expressed as the mean $\pm S D, n=10$. $\otimes P<0.05$, $\triangle \otimes \otimes P<0.01$. 


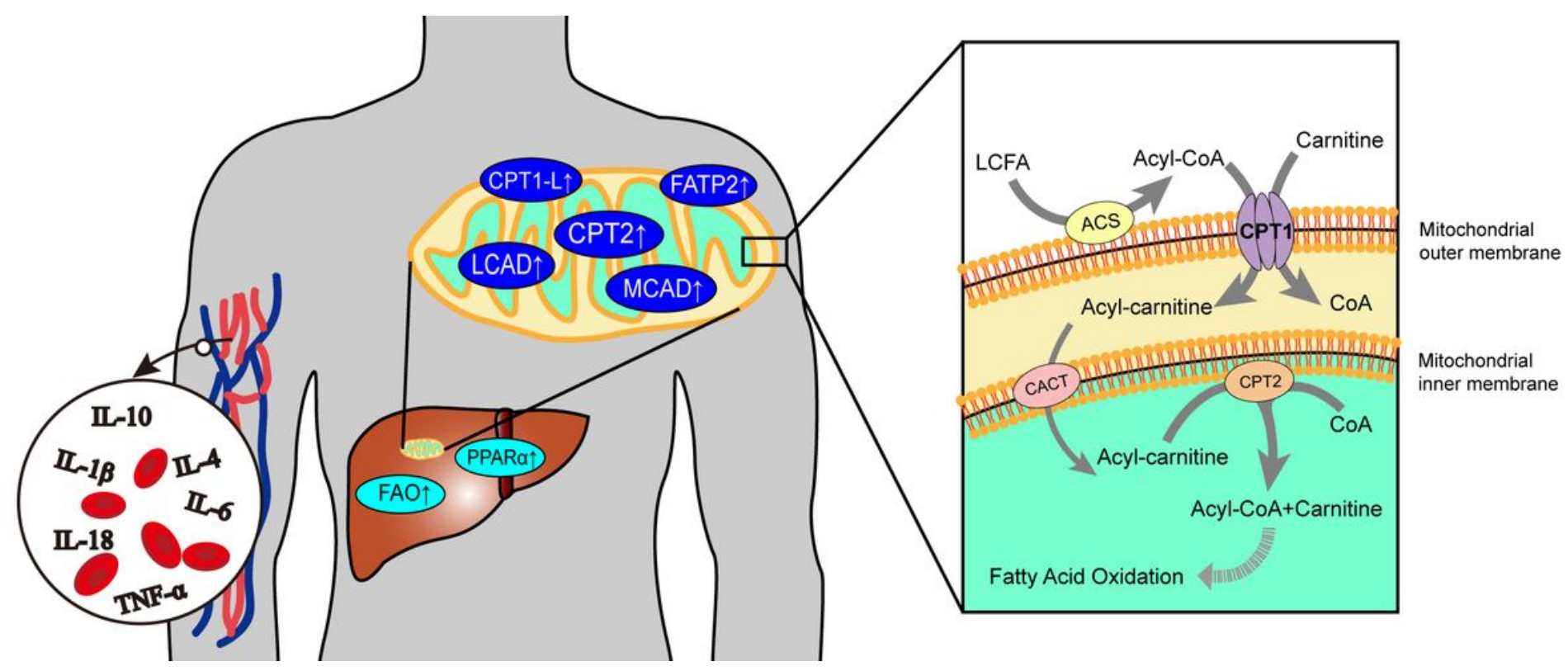

Figure 6

Carnitine 0-palmitoyltransferase 1 is anchored on the mitochondrial outer membranes and takes charge of converting acyl-CoAs into acylcarnitines that are shuttled across the mitochondria membranes by the translocase and converted back to acyl-CoAs by carnitine 0-palmitoyltransferase 2 inside mitochondria before undergoing fatty acid oxidation. Increasing the expression of PPARa, CPT1-L, CPT2, MCAD, LCAD and FATP2 enhances the capacity of hepatocytes to mediate $\beta$-oxidation of fatty acids and minimizes lipid accumulation and inflammation response, which results in NAFLD.

\section{Supplementary Files}

This is a list of supplementary files associated with this preprint. Click to download.

- WesternB.pdf 